\title{
GENERALIZED ELLIPTIC INTEGRALS
}

\author{
Ville Heikkala \\ Mavina K. Vamanamurthy, \\ and \\ Matti Vuorinen \\ File: ggem73.tex, printed: 2019-2-23, 2.29
}

\begin{abstract}
Jacobi's elliptic integrals and elliptic functions arise naturally from the SchwarzChristoffel conformal transformation of the upper half plane onto a rectangle. In this paper we study generalized elliptic integrals which arise from the analogous mapping of the upper half plane onto a quadrilateral and obtain sharp monotonicity and convexity properties for certain combinations of these integrals, thus generalizing analogous well-known results for classical conformal capacity and quasiconformal distortion functions.
\end{abstract}

2000 Mathematics Subject Classification: Primary 33B15, 33C05, Secondary $30 \mathrm{C} 62$.

\section{Introduction}

Given complex numbers $a, b$, and $c$ with $c \neq 0,-1,-2, \ldots$, the Gaussian hypergeometric function is the analytic continuation to the slit plane $\mathbb{C} \backslash[1, \infty)$ of the series

$$
F(a, b ; c ; z)={ }_{2} F_{1}(a, b ; c ; z)=\sum_{n=0}^{\infty} \frac{(a, n)(b, n)}{(c, n)} \frac{z^{n}}{n !},|z|<1 .
$$

Here $(a, 0)=1$ for $a \neq 0$, and $(a, n)$ is the shifted factorial function or the Appell symbol

$$
(a, n)=a(a+1)(a+2) \cdots(a+n-1)
$$

for $n \in \mathbb{N} \backslash\{0\}$, where $\mathbb{N}=\{0,1,2, \ldots\}$.

A generalized modular equation of order (or degree) $p>0$ is

$$
\frac{F\left(a, b ; c ; 1-s^{2}\right)}{F\left(a, b ; c ; s^{2}\right)}=p \frac{F\left(a, b ; c ; 1-r^{2}\right)}{F\left(a, b ; c ; r^{2}\right)}, 0<r<1 .
$$

\footnotetext{
$\dagger^{\dagger}$ Author supported by the Magnus Ehrnrooth fund of the Finnish Academy of Science and Letters.
} 
Sometimes we just call this an $(a, b, c)$-modular equation of order $p$ and we usually assume that $a, b, c>0$ with $a+b \geq c$, in which case this equation uniquely defines $s$, see Lemma 4.5 .

Many particular cases of (1.2) have been studied in the literature on both analytic number theory and geometric function theory, [Be, $[\mathrm{BB},[\mathrm{BBG}$, , AVV], AQVV], [LV]. The classical case $(a, b, c)=\left(\frac{1}{2}, \frac{1}{2}, 1\right)$ was studied already by Jacobi and many others in the nineteenth century, see [Be. In 1995 B. Berndt, S. Bhargava, and F. Garvan published an important paper $[\mathrm{BBG}]$ in which they studied the case $(a, b, c)=(a, 1-a, 1)$ and $p$ an integer. For several rational values of $a$ such as $a=\frac{1}{3}, \frac{1}{4}, \frac{1}{6}$ and integers $p$ (e.g. $p=2,3,5,7,11, \ldots)$ they were able to give proofs for numerous algebraic identities stated by Ramanujan in his unpublished notebooks. These identities involve $r$ and $s$ from (1.2). After the publication of [BBG] many papers have appeared on modular equations, see e.g. [AQVV], [Be, [BCKZ], [CLT], Q], and [S].

To rewrite (1.2) in a slightly shorter form, we use the decreasing homeomorphism $\mu_{a, b, c}:(0,1) \rightarrow(0, \infty)$, defined by

$$
\mu_{a, b, c}(r)=\frac{B(a, b)}{2} \frac{F\left(a, b ; c ; r^{\prime 2}\right)}{F\left(a, b ; c ; r^{2}\right)}, r \in(0,1)
$$

for $a, b, c>0, a+b \geq c$, where $B$ is the beta function, see (3.5) below. We call $\mu_{a, b, c}$ the generalized modulus, cf. [LV, (2.2)]. We can now write (1.2) as

$$
\mu_{a, b, c}(s)=p \mu_{a, b, c}(r), 0<r<1 .
$$

With $p=1 / K, K>0$, the solution of (1.2) is then given by

$$
s=\varphi_{K}^{a, b, c}(r)=\mu_{a, b, c}^{-1}\left(\mu_{a, b, c}(r) / K\right) .
$$

We call $\varphi_{K}^{a, b, c}$ the $(a, b, c)$-modular function with degree $p=1 / K$ [BBG], AQVV, (1.5)].

In the case $a<c$ we also use the notation

$$
\mu_{a, c}=\mu_{a, c-a, c}, \quad \varphi_{K}^{a, c}=\varphi_{K}^{a, c-a, c} .
$$

For $0<a<\min \{c, 1\}$ and $0<b<c \leq a+b$, define the generalized complete elliptic integrals of the first and second kinds (cf. AQVV, (1.9), (1.10), (1.3), and (1.5)]) on [0,1] by

$$
\begin{gathered}
\mathcal{K}=\mathcal{K}_{a, b, c}=\mathcal{K}_{a, b, c}(r)=\frac{B(a, b)}{2} F\left(a, b ; c ; r^{2}\right), \\
\mathcal{E}=\mathcal{E}_{a, b, c}=\mathcal{E}_{a, b, c}(r)=\frac{B(a, b)}{2} F\left(a-1, b ; c ; r^{2}\right), \\
\mathcal{K}^{\prime}=\mathcal{K}_{a, b, c}^{\prime}=\mathcal{K}_{a, b, c}\left(r^{\prime}\right), \text { and } \mathcal{E}^{\prime}=\mathcal{E}_{a, b, c}^{\prime}=\mathcal{E}_{a, b, c}\left(r^{\prime}\right)
\end{gathered}
$$

for $r \in(0,1), r^{\prime}=\sqrt{1-r^{2}}$. The end values are defined by limits as $r$ tends to $0+$ and $1-$, respectively. In particular, we denote $\mathcal{K}_{a, c}=\mathcal{K}_{a, c-a, c}$ and $\mathcal{E}_{a, c}=\mathcal{E}_{a, c-a, c}$. Thus, by (3.9) below,

$$
\mathcal{K}_{a, b, c}(0)=\mathcal{E}_{a, b, c}(0)=\frac{B(a, b)}{2}
$$


and

$$
\mathcal{E}_{a, b, c}(1)=\frac{1}{2} \frac{B(a, b) B(c, c+1-a-b)}{B(c+1-a, c-b)}, \mathcal{K}_{a, b, c}(1)=\infty .
$$

Note that the restrictions on $a, b$ and $c$ ensure that the function $\mathcal{K}_{a, b, c}$ is increasing and unbounded whereas $\mathcal{E}_{a, b, c}$ is decreasing and bounded, as in the classical case $a=b=$ $\frac{1}{2}, c=1$. Note also that our terminology differs from that of [BB, Section 5.5], where generalized elliptic integrals refer to the particular case $c=1$.

In this paper we study the modular function $\varphi_{K}^{a, b, c}$ and the generalized modulus $\mu_{a, b, c}$ as well as the generalized elliptic integrals $\mathcal{K}_{a, b, c}$ and $\mathcal{E}_{a, b, c}$. In the case $b=1-a, c=1$, these functions coincide with the special cases $\varphi_{K}^{a}, \mu_{a}, \mathcal{K}_{a}$, and $\mathcal{E}_{a}$ which were studied in AQVV].

In Section 2 we construct a conformal mapping from a quadrilateral with internal angles $b \pi,(c-b) \pi,(1-a) \pi$, and $(1-c+a) \pi$ onto the upper half plane. We denote this mapping by $\mathrm{sn}_{a, b, c}$. If $b=1-a$ and $c=1$, this mapping reduces to the generalized elliptic sine function $\mathrm{sn}_{a}$ in AQVV, 2.1]. In a later work, we study the dependence of the modulus on the geometry of the quadrilateral, [DV], [RV].

In Section 3 we recall some basic properties of the hypergeometric, gamma, and beta functions, that are used in the sequel.

Section 4 contains our main results: differentiation formulas and monotone properties of the generalized elliptic integrals and of several combinations of these functions. Theorems 4.3 and 4.4, which provide sufficient conditions for the quotients of two Maclaurin series to be monotone increasing, seem to be of independent interest and, as far as we know, these results are new. Also a similar result is given for the quotient of two polynomials of the same degree.

Throughout this paper we denote $r^{\prime}=\sqrt{1-r^{2}}$ whenever $r \in(0,1)$. The standard symbols $\mathbb{C}, \mathbb{R}, \mathbb{Z}$, and $\mathbb{N}$ denote the sets of complex numbers, real numbers, integers, and natural numbers (with zero included), respectively.

\section{The Schwarz-Christoffel map onto a quadrilateral}

For $0<a, b<1, \max \{a+b, 1\} \leq c \leq \min \{a, b\}+1, r \in(0,1)$, let $g_{r}(t)=t^{b-1}(1-$ $t)^{c-b-1}\left(1-r^{2} t\right)^{-a}, t \in \mathbb{C}, \operatorname{Im} t \geq 0$, denote the analytic branch for which the argument of each of the factors $t, 1-t$, and $1-r^{2} t$ is $\pi$ whenever it is real and negative. Denote $C=C(b, c)=1 / B(b, c-b)$. We define the generalized Jacobi sine function $\operatorname{sn}_{a, b, c}(w)=$ $\operatorname{sn}_{a, b, c}(w, r)$ as the inverse of the function $f$ given on the closed upper half plane by

$$
\begin{aligned}
w=f(z)=f_{a, b, c}(z) & =C \int_{0}^{z} g_{r}(t) d t \\
& =C \int_{0}^{z} t^{b-1}(1-t)^{c-b-1}\left(1-r^{2} t\right)^{-a} d t \\
& =e^{i(a+b+1-c) \pi} C r^{-2 a} \int_{0}^{z} t^{b-1}(t-1)^{c-b-1}\left(t-1 / r^{2}\right)^{(1-a)-1} d t .
\end{aligned}
$$

Recall the Euler integral representation [AAR, Theorem 2.2.1] [AS, 15.3.1]

$$
F(a, b ; c ; z)=\frac{\Gamma(c)}{\Gamma(b) \Gamma(c-b)} \int_{0}^{1} t^{b-1}(1-t)^{c-b-1}(1-t z)^{-a} d t
$$




$$
=C(b, c) e^{i(a+b+1-c) \pi} \int_{0}^{1} t^{b-1}(t-1)^{c-b-1}(t z-1)^{-a} d t
$$

for $\operatorname{Re} c>\operatorname{Re} b>0$ and $z \in \mathbb{C} \backslash\{u \in \mathbb{R} \mid u \geq 1\}$.

The next result is well-known, but for the sake of completeness a proof is given. As general references concerning the Schwarz-Christoffel mapping [M] and [N] may be mentioned.

2.3. Proposition. The Schwarz-Christoffel mapping of the upper half plane H onto a quadrilateral with turning angles $\beta_{k} \pi$ at the vertices $a_{k} \in \mathbb{R}$ is

$$
w=F(z)=\int_{0}^{z} \frac{d z}{\Pi_{k=1}^{4}\left(z-a_{k}\right)^{\beta_{k}}}, \quad-1<\beta_{k}<1, \sum_{k=1}^{4} \beta_{k}=2,
$$

where $a_{k}$ are the points on the real axis that $F$ maps to the four vertices of the quadrilateral.

Proof. As in [N, pp. 192-193] let $T: H \rightarrow U$ be the Möbius transformation $T(z)=$ $(z-i) /(z+i)$, where $U$ is the unit disk $\{z:|z|<1\}$. Then $f(z)=F\left(T^{-1}(z)\right)$ maps $U$ onto the quadrilateral $Q$ and

$$
f(\zeta)=\int_{0}^{\zeta} \frac{d w}{\Pi_{k=1}^{4}\left(w-\zeta_{k}\right)^{\beta_{k}}}
$$

where $\zeta_{k}$ are the points on the unit circle that map onto the four vertices of the quadrilateral $Q$. Now

$$
\frac{\zeta f^{\prime \prime}(\zeta)}{f^{\prime}(\zeta)}=-\sum_{k=1}^{4} \beta_{k} \frac{\zeta}{\left(\zeta-\zeta_{k}\right)}=\sum_{k=1}^{4} \beta_{k} \frac{1+\overline{\zeta_{k}} \zeta}{1-\overline{\zeta_{k}} \zeta}
$$

and recalling that the $\beta_{k}$ sum to 2 we have that

$$
1+\frac{\zeta f^{\prime \prime}(\zeta)}{f^{\prime}(\zeta)}=\frac{1}{2} \sum_{k=1}^{4} \beta_{k} \frac{1+\overline{\zeta_{k}} \zeta}{1-\overline{\zeta_{k}} \zeta}
$$

For all points $\zeta \in U$

$$
\operatorname{Re}\left\{1+\frac{\zeta f^{\prime \prime}(\zeta)}{f^{\prime}(\zeta)}\right\}=\frac{1}{2} \sum_{k=1}^{4} \beta_{k} \operatorname{Re}\left\{\frac{1+\overline{\zeta_{k}} \zeta}{1-\overline{\zeta_{k}} \zeta}\right\}=\frac{1}{2} \sum_{k=1}^{4} \beta_{k} \frac{1-|\zeta|^{2}}{\left|1-\overline{\zeta_{k}} \zeta\right|^{2}}>0 .
$$

This proves that $f$ maps $U$ bijectively onto a convex domain, since $\operatorname{Re}\left\{1+\zeta f^{\prime \prime}(\zeta) / f^{\prime}(\zeta)\right\}>$ 0 is a necessary and sufficient condition for this to be true, see [N, Ex. 5, p. 224].

2.4. Theorem. Let $H$ denote the closed upper half-plane $\{z \in \mathbb{C} \mid \operatorname{Im} z \geq 0\}$ and let $0<a, b<1, \max \{a+b, 1\} \leq c \leq 1+\min \{a, b\}, r \in(0,1)$. Then the function $f$ in (2.1) is a homeomorphism of $H$ onto the quadrilateral $Q$ with vertices

$$
f(0)=0, f(1)=F\left(a, b ; c ; r^{2}\right),
$$



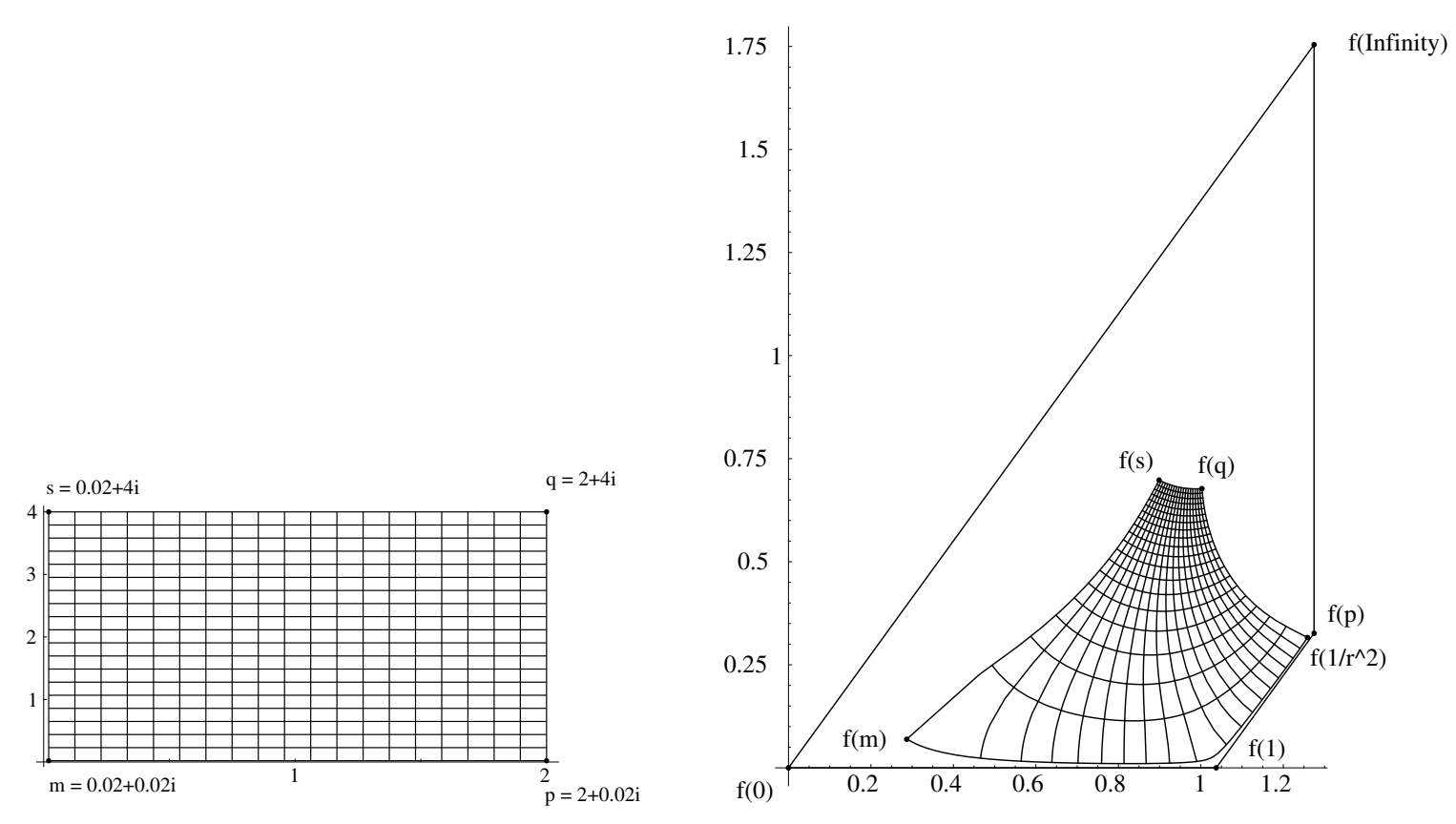

Figure 1: The image quadrilateral and the image of a grid under the mapping $f_{a, b, c}$ with $a=0.2, b=0.3, c=1.0, r=0.7$

$$
f\left(1 / r^{2}\right)=f(1)+\frac{B(c-b, 1-a)}{B(b, c-b)} e^{(b+1-c) i \pi}{r^{\prime 2(c-a-b)}} F\left(c-a, c-b ; c+1-a-b ; r^{\prime 2}\right),
$$

and

$f(\infty)=f\left(1 / r^{2}\right)+\frac{B(1-a, a+1-c)}{B(b, c-b)} e^{i(a+b+1-c) \pi} r^{2(1-c)} r^{2(c-a-b)} F\left(1-b, 1-a ; 2-c ; r^{2}\right)$,

and interior angles $b \pi,(c-b) \pi,(1-a) \pi$ and $(a+1-c) \pi$, respectively, at these vertices. It is conformal in the interior of $H$.

Proof. It is clear that $f(0)=0$ and by (2.2) $f(1)=F\left(a, b ; c ; r^{2}\right)$. Next we evaluate

$$
\begin{aligned}
f\left(1 / r^{2}\right) & =C \int_{0}^{1 / r^{2}} g_{r}(t) d t \\
& =C \int_{0}^{1} g_{r}(t) d t+C \int_{1}^{1 / r^{2}} g_{r}(t) d t \\
& =F\left(a, b ; c ; r^{2}\right)+C \int_{1}^{1 / r^{2}} g_{r}(t) d t .
\end{aligned}
$$

To evaluate the second integral above, we make the change of variable $t=1 /\left(1-r^{\prime 2} u\right)$ for which $d t=\left(1-r^{\prime 2} u\right)^{-2} r^{\prime 2} d u$. We observe that this change of variable is simply the restriction to reals of the plane Möbius transformation taking the ordered triple $\left(1,1 / r^{2}, 0\right)$ onto the ordered triple $(0,1, \infty)$. Then

$$
g_{r}(t) d t=\left(1-{r^{\prime}}^{2} u\right)^{1-b}\left(1-\frac{1}{1-r^{\prime 2} u}\right)^{c-b-1}\left(1-\frac{r^{2}}{1-r^{\prime 2} u}\right)^{-a}\left(1-{r^{\prime}}^{2} u\right)^{-2}{r^{\prime}}^{2} d u
$$




$$
\begin{aligned}
& =(-1)^{b+1-c}{r^{2(c-a-b)}}^{c-b-1}(1-u)^{-a}\left(1-{r^{\prime}}^{2} u\right)^{a-c} d u \\
& =e^{(b+1-c) i \pi} r^{\prime 2(c-a-b)} u^{c-b-1}(1-u)^{-a}\left(1-{r^{\prime}}^{2} u\right)^{a-c} d u
\end{aligned}
$$

and by (2.2) we get

$$
\begin{aligned}
C \int_{1}^{1 / r^{2}} g_{r}(t) d t= & e^{(b+1-c) i \pi} r^{2(c-a-b)} \frac{\Gamma(c)}{\Gamma(b) \Gamma(c-b)} \int_{0}^{1} u^{c-b-1}(1-u)^{-a}\left(1-r^{2} u\right)^{a-c} d u \\
= & e^{(b+1-c) i \pi} r^{2(c-a-b)} \frac{\Gamma(c)}{\Gamma(b) \Gamma(c-b)} \frac{\Gamma(c-b) \Gamma(1-a)}{\Gamma(c+1-a-b)} \\
& \cdot F\left(c-a, c-b ; c+1-a-b ; r^{\prime 2}\right) \\
= & \frac{B(c-b, 1-a)}{B(b, c-b)} e^{(b+1-c) i \pi} r^{2(c-a-b)} F\left(c-a, c-b ; c+1-a-b ; r^{2}\right) \\
= & \frac{B(c-b, 1-a)}{B(b, c-b)} e^{(b+1-c) i \pi} r^{2(1-c)}{r^{\prime 2(c-a-b)}}^{2\left(1-a, 1-b ; c+1-a-b ; r^{\prime 2}\right)}
\end{aligned}
$$

where the last expression follows from (3.9) below. Hence $f\left(1 / r^{2}\right)$ has the value claimed. We proceed to evaluate the remaining value, namely

$$
f(\infty)=C \int_{0}^{\infty} g_{r}(t) d t=C \int_{0}^{1 / r^{2}} g_{r}(t) d t+C \int_{1 / r^{2}}^{\infty} g_{r}(t) d t
$$

The first integral above equals $f\left(1 / r^{2}\right)$. To compute the second one, we apply the change of variable $t=\left(1-r^{2} v\right) /\left(r^{2}(1-v)\right)$. We observe that this change of variable is simply the restriction to reals of the plane Möbius transformation taking the ordered triple $\left(1 / r^{2}, \infty, 1\right)$ onto the ordered triple $(0,1, \infty)$. Then $d t=\left(1-r^{2}\right) /\left(r^{2}(1-v)^{2}\right) d v, t=1 / r^{2}$ gives $v=0$, and $t=\infty$ gives $v=1$. We get

$$
\begin{aligned}
g_{r}(t) d t= & \left(\frac{1-r^{2} v}{r^{2}(1-v)}\right)^{b-1}\left(1-\frac{1-r^{2} v}{r^{2}(1-v)}\right)^{c-b-1} \cdot \\
& \cdot\left(1-\frac{r^{2}\left(1-r^{2} v\right)}{r^{2}(1-v)}\right)^{-a}\left(\frac{1-r^{2}}{r^{2}(1-v)^{2}}\right) d v \\
= & r^{2(1+a-b+b+1)}(1-v)^{a-c}\left(1-r^{2} v\right)^{b-1}\left(r^{2}-1\right)^{c-b-1}\left(r^{2}\left(r^{2}-1\right) v\right)^{-a}\left(1-r^{2}\right) d v \\
= & (-1)^{a+b+1-c} r^{2(c-a-b)} r^{2(1-c)} v^{-a}(1-v)^{a-c}\left(1-r^{2} v\right)^{b-1} d v
\end{aligned}
$$

so that

$$
\begin{aligned}
C \int_{1 / r^{2}}^{\infty} g_{r}(t) d t= & (-1)^{a+b+1-c} r^{\prime 2(c-a-b)} r^{2(1-c)} C \int_{0}^{1} v^{-a}(1-v)^{a-c}\left(1-r^{2} v\right)^{b-1} d v \\
= & (-1)^{a+b+1-c} r^{2(c-a-b)} r^{2(1-c)} \frac{B(1-a, 1+a-c)}{B(b, c-b)} . \\
& \cdot F\left(1-b, 1-a ; 2-c ; r^{2}\right) .
\end{aligned}
$$

The claimed value for $f(\infty)$ follows. 
It follows from the formula (2.1) that (see e.g. [M, pp. 128-134]) $f$ is a SchwarzChristoffel transformation which maps $H$ onto a quadrilateral $Q$ with vertices $f(0), f(1)$, $f\left(1 / r^{2}\right)$, and $f(\infty)$ and interior angles $b \pi,(c-b) \pi,(1-a) \pi$, and $(1-c+a) \pi$ in counterclockwise order. By Proposition $2.3 f$ is univalent.

2.5. Corollary. Let $0<a, b<1, \max \{a+b, 1\} \leq c \leq 1+\min \{a, b\}$, and let $Q$ be a quadrilateral in the upper half plane with vertices $0,1, A$ and $B$, the interior angles at which are, respectively, $b \pi,(c-b) \pi,(1-a) \pi$ and $(1+a-c) \pi$. Then the conformal modulus of $Q$ (cf. $[L V])$ is given by

$$
\bmod (Q)=\mathcal{K}\left(r^{\prime}\right) / \mathcal{K}(r),
$$

where $r \in(0,1)$ satisfies the equation

$$
A-1=\frac{L r^{2(c-a-b)} F\left(c-a, c-b ; c+1-a-b ; r^{\prime 2}\right)}{F\left(a, b ; c ; r^{2}\right)}=G(r),
$$

say, and

$$
L=\frac{B(c-b, 1-a)}{B(b, c-b)} e^{(b+1-c) i \pi} .
$$

Proof. Clearly, $\arg (A-1)=(b+1-c) \pi=\arg (L)$. Since $G(0)=\infty$ and $G(1)=0$, it follows that a unique solution $r \in(0,1)$ of equation (2.6) exists. Let $f$ be as in Theorem 2.4 and let $g=f / f(1)$. Then $g$ maps the upper half plane $H$ onto $Q$, with $g(0)=0, g(1)=1, g\left(1 / r^{2}\right)=A$, and $g(\infty)=B$. The function $h=\mathrm{sn}^{-1}$ maps the first quadrant conformally [Bo] onto the standard rectangle $R$, with $h(0)=0, h(1)=\mathcal{K}(r)$, $h(1 / r)=\mathcal{K}(r)+i \mathcal{K}\left(r^{\prime}\right)$, and $h(\infty)=i \mathcal{K}\left(r^{\prime}\right)$. Hence the function $k=h(\sqrt{ })$ maps $H$ conformally onto $R$. Thus, by conformal invariance, $\bmod (Q)=\mathcal{K}\left(r^{\prime}\right) / \mathcal{K}(r)$.

2.7. Remark. (1) The quadrilateral $Q$ in Theorem 2.4 reduces to a trapezoid if and only if $c=1$ or $c=a+b$, to a parallelogram if and only if $c=1=a+b$, AQVV and to a rectangle (the classical case) if and only if $a=b=\frac{1}{2}$ and $c=1,[\mathrm{BO}]$.

(2) The hypotheses in Corollary 2.5 imposed on the triple $a, b, c$ imply that the quadrilateral $Q$ is convex.

2.8. Remark. Bowman [Bo, pp. 103-104] gives a formula for the conformal modulus of the quadrilateral with vertices $0,1,1+h i$, and $(h-1) i$ when $h>1$ as $q=\mathcal{K}(r) / \mathcal{K}\left(r^{\prime}\right)$ where

$$
r=\left(\frac{t_{1}-t_{2}}{t_{1}+t_{2}}\right)^{2}, \quad t_{1}=\mu^{-1}\left(\frac{\pi}{2 c}\right), \quad t_{2}=\mu^{-1}\left(\frac{\pi c}{2}\right), \quad c=2 h-1 .
$$

Therefore, the quadrilateral can be conformally mapped onto the rectangle $0,1,1+q i$, $q i$ with vertices corresponding. 
2.9. Computational discovery. We have written a Mathematica ${ }^{\circledR}$ function that computes the modulus of the quadrilateral with vertices at $0,1, A, B$ where $\operatorname{Im} A>$ $0, \operatorname{Im} B>0$. This led to the following discovery for symmetric quadrilaterals:

$$
\text { If }|B|=1 \text { and } 2 \arg A=\arg B \text {, then the modulus is equal to } 1 \text {. }
$$

It is not difficult to prove this analytically (see e.g. [Hen, p. 433] or [Her]). The following figure illuminates the variation of the modulus of the quadrilateral with vertices $0,1, x+$ $i y, i$ in the first quadrant.

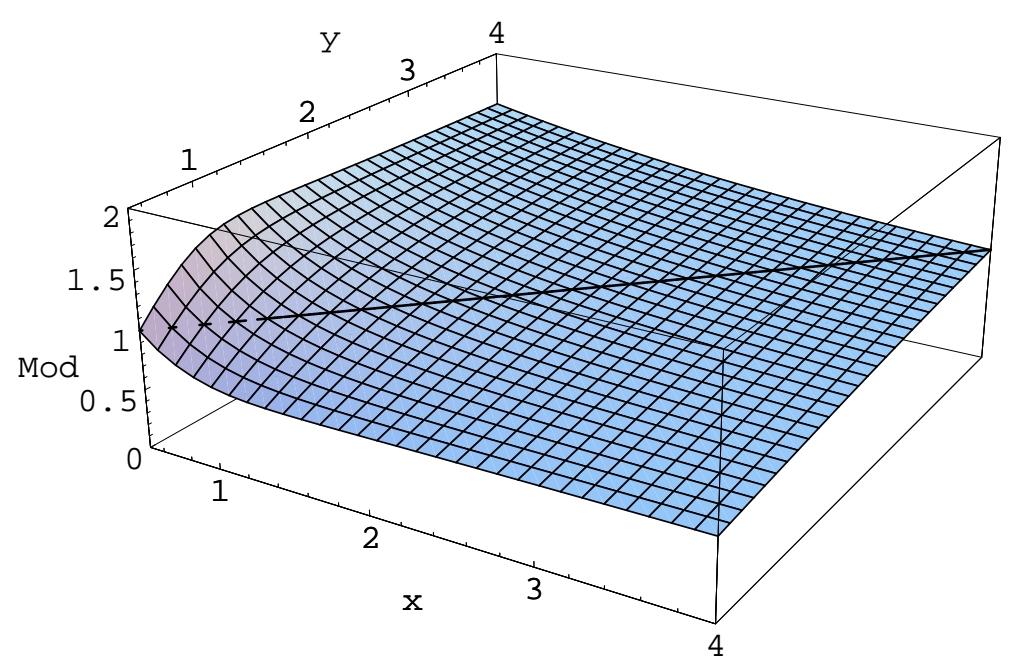

Figure 2: Modulus of the quadrilateral with vertices $0,1, x+i y, i$ and the line $(x, x, 1)$.

2.11. Duplication formula for quadrilateral modulus. For the sake of convenient notation, denote by $\operatorname{QM}(a, b, c, d)$ the modulus of the polygon with vertices $a, b, c, d$. That is, $\operatorname{QM}(a, b, c, d)=q$ if and only if there exists a conformal map of the polygon onto the rectangle with vertices $1+i q, i q, 0,1$ such that the vertices correspond. Clearly $\mathrm{QM}(1+$ $i q, i q, 0,1)=q$. Remark 2.8 gives now an explicit formula for $\mathrm{QM}(1+i h, i(h-1), 0,1)$ when $h>1$. (By the way, it seems likely that $\mathrm{QM}(1+i h, i(h-1), 0,1) \in[h-1, h]$.) By (2.10) we see that $\mathrm{QM}\left(t e^{i \pi / 4}, i, 0,1\right)=1$ for all $t>0$ and it is clear by symmetry that

$$
\mathrm{QM}\left(t e^{i(\pi / 4-\alpha)}, i, 0,1\right)=1 / \mathrm{QM}\left(t e^{i(\pi / 4+\alpha)}, i, 0,1\right)
$$

for all $\alpha \in(0, \pi / 4)$ and all $t>0$. From [AQVV, Corollary 2.3] we have an expression for the parallelogram case $\mathrm{QM}\left(1+r e^{i \alpha}, r e^{i \alpha}, 0,1\right)$.

We are not familiar with cases other than those mentioned above where the values of $\mathrm{QM}(a, b, c, d)$ could be expressed in reasonably simple form. Therefore it might be of some interest to record a duplication formula for $\operatorname{QM}(a, b, c, d)$ which follows from an elementary symmetry consideration. To this end, let $h, k>0$ and consider the quadrilaterals $(1+$ $i h, i k,-i k, 1-i h)$ and $(1+i h, i k, 0,1)$. It is clear by symmetry that

$$
\mathrm{QM}(1+i h, i k,-i k, 1-i h)=2 \mathrm{QM}(1+i h, i k, 0,1) .
$$


We now use the invariance of $\operatorname{QM}(a, b, c, d)$ under homotheties to express this result in a simpler form. Choose a homothety $T(z)=a z+b$ with $T(-i k)=0, T(1-i h)=1$. Then $a=1 / c, b=i k / c$ with $c=1+i(k-h)$. Because

$$
T(1+i h)=(1+i(h+k)) / c, T(i k)=2 i k / c, T(-i k)=0, \text { and } T(1-i h)=1,
$$

we have for $h, k>0, c=1+i(k-h)$ that

$$
\mathrm{QM}((1+i(h+k)) / c, 2 i k / c, 0,1)=2 \mathrm{QM}(1+i h, i k, 0,1) .
$$

\begin{tabular}{|c|c|c|c|c|c|}
\hline $\mathrm{m} \backslash \mathrm{n}$ & 1 & 2 & 3 & 4 & 5 \\
\hline 1 & 1.000000 & 1.279261 & 1.354244 & 1.383086 & 1.397799 \\
2 & 0.781700 & 1.000000 & 1.127663 & 1.201627 & 1.248066 \\
3 & 0.738419 & 0.886789 & 1.000000 & 1.080783 & 1.138566 \\
4 & 0.723020 & 0.832204 & 0.925254 & 1.000000 & 1.058739 \\
5 & 0.715410 & 0.801239 & 0.878297 & 0.944519 & 1.000000 \\
\hline
\end{tabular}

Table 1: Numerical values of $\operatorname{QM}(m+i n, i, 0,1)$ for $m, n=1, \ldots, 5$. The values have been truncated to six decimal places

The dependence of the modulus of a polygonal quadrilateral on the geometry is the subject of our subsequent work [DV], [RV].

\section{Hypergeometric functions}

Let $\Gamma$ denote Euler's gamma function and let $\Psi$ be its logarithmic derivative (also called the digamma function), $\Psi(z)=\Gamma^{\prime}(z) / \Gamma(z)$. By [Ah, p. 198] the function $\Psi$ and its derivative have the series expansions

$$
\Psi(z)=-\gamma-\frac{1}{z}+\sum_{n=1}^{\infty} \frac{z}{n(n+z)}, \quad \Psi^{\prime}(z)=\sum_{n=0}^{\infty} \frac{1}{(n+z)^{2}},
$$

where $\gamma=-\Psi(1)=\lim _{n \rightarrow \infty}\left(\sum_{k=1}^{n} 1 / k-\log n\right)=0.57721 \ldots$ is the Euler-Mascheroni constant. From (3.1) it is seen that $\Psi$ is strictly increasing on $(0, \infty)$ and that $\Psi^{\prime}$ is strictly decreasing there, so that $\Psi$ is concave. Moreover, $\Psi(z+1)=\Psi(z)+1 / z$ and $\Psi\left(\frac{1}{2}\right)=-\gamma-2 \log 2$, see [AS, Ch. 6].

For all $z \in \mathbb{C} \backslash\{0,-1,-2, \ldots\}$ and for all $n \in \mathbb{N}$ we have

$$
\Gamma(z+n)=(z, n) \Gamma(z),
$$

a fact which follows by induction [WW, 12.12]. This enables us to extend the Appell symbol for all complex values of $a$ and $a+t$, except for non-positive integer values, by

$$
(a, t)=\frac{\Gamma(a+t)}{\Gamma(a)} .
$$


Furthermore, the gamma function satisfies the reflection formula [WW, 12.14]

$$
\Gamma(z) \Gamma(1-z)=\frac{\pi}{\sin (\pi z)}
$$

for all $z \notin \mathbb{Z}$. In particular, $\Gamma\left(\frac{1}{2}\right)=\sqrt{\pi}$.

The beta function is defined for $\operatorname{Re} x>0$, Re $y>0$ by

$$
B(x, y)=\int_{0}^{1} t^{x-1}(1-t)^{y-1} d t=\frac{\Gamma(x) \Gamma(y)}{\Gamma(x+y)} .
$$

We use the standard notation for contiguous hypergeometric functions (cf. $[\mathrm{R}]$ )

$$
F=F(a, b ; c ; z), F(a+)=F(a+1, b ; c ; z), \quad F(a-)=F(a-1, b ; c ; z),
$$

etc. We also let

$v=v(z)=F, u=u(z)=F(a-), v_{1}=v_{1}(z)=v(1-z)$, and $u_{1}=u_{1}(z)=u(1-z)$.

The derivative of $F$ can be written in the following several different forms which will be useful in deriving the fifteen important Gauss contiguous relations $\mathrm{R}$.

$$
\begin{aligned}
\frac{d v}{d z}=\frac{d F}{d z} & =\frac{a}{z}(F(a+)-F)=\frac{b}{z}(F(b+)-F)=\frac{c-1}{z}(F(c-)-F) \\
& =\frac{a b}{c} F(a+, b+; c+)=\frac{1}{1-z}\left((a+b-c) F+\frac{(c-a)(c-b)}{c} F(c+)\right) \\
& =\frac{(c-a) u+(a-c+b z) v}{z(1-z)}
\end{aligned}
$$

and

$$
\frac{d u}{d z}=\frac{d F(a-)}{d z}=(a-1)\left(F+\frac{b-c}{c} F(c+)\right)=\frac{a-1}{z}(v-u) .
$$

In particular, from (3.6) it follows that (cf. AQVV, Theorem 3.12 (3)])

$$
\frac{a b}{c} z(1-z) F(a+1, b+1 ; c+1 ; z)=(c-a) u(z)+(a-c+b z) v(z) .
$$

The behavior of the hypergeometric function near $z=1$ in the three cases $\operatorname{Re}(a+b-$ $c)<0, a+b=c$, and $\operatorname{Re}(a+b-c)>0$, respectively, is given by

$$
\left\{\begin{array}{l}
F(a, b ; c ; 1)=\frac{\Gamma(c) \Gamma(c-a-b)}{\Gamma(c-a) \Gamma(c-b)}, \\
B(a, b) F(a, b ; a+b ; z)+\log (1-z)=R(a, b)+\mathrm{O}((1-z) \log (1-z)), \\
F(a, b ; c ; z)=(1-z)^{c-a-b} F(c-a, c-b ; c ; z),
\end{array}\right.
$$

where $R(a, b)=-\Psi(a)-\Psi(b)-2 \gamma$. The above asymptotic formula for the zero-balanced case $a+b=c$ is due to Ramanujan (see [Ask], Be]). This formula is implied by [AS, 15.3.10]. Note that $R\left(\frac{1}{2}, \frac{1}{2}\right)=\log 16$. 
For complex $a, b, c$, and $z$, with $|z|<1$, we let

$$
\mathcal{M}(z)=\mathcal{M}(a, b, c, z)=z(1-z)\left(v_{1}(z) \frac{d v}{d z}-v(z) \frac{d v_{1}}{d z}\right) .
$$

From (3.6) it is easy to see that

$$
\begin{aligned}
\mathcal{M} & =(c-a)\left(u v_{1}+u_{1} v\right)+(2(a-c)+b) v v_{1} \\
& =(c-a)\left(u v_{1}+u_{1} v-v v_{1}\right)+(a+b-c) v v_{1} .
\end{aligned}
$$

It follows from AQVV, Corollary 3.13(5)] and (3.4) that

$$
\mathcal{M}(a, 1-a, 1, r)=\frac{1-a}{\Gamma(a) \Gamma(2-a)}=\frac{\sin (\pi a)}{\pi}
$$

for $0<a<1$ and $0 \leq r<1$. In particular, we get the classical Legendre relation ([AAR], [BF])

$$
\mathcal{M}(1 / 2,1 / 2,1, r)=\frac{1}{\pi} .
$$

The next result generalizes AQVV, Theorem 3.9].

3.14. Theorem. For $0<a, b<c$, let the function $f$ be defined on $[0, \infty)$ by $f(x)=$ $F\left(a, b ; c ; 1-e^{-x}\right)$ and let $g(x)=f(x) \exp (-(a+b-c) x)$. Then $f$ and $g$ are increasing, with $f(0)=g(0)=1$. If $a+b>c$, then $f(\infty)=\infty$. If $a+b=c$, then $f(\infty)=g(\infty)=\infty$. If $a+b<c$, then $f(\infty)=B(c, c-a-b) / B(c-a, c-b)$ and $g(\infty)=\infty$. Moreover, $h(x)=f^{\prime}(x) e^{-x(a+b-c)}$ is also increasing, with $h(0)=a b / c$ and $h(\infty)=\Gamma(c) \Gamma(a+b+1-$ $c) /(\Gamma(a) \Gamma(b))$ or $h(\infty)=\infty$ according as $a+b+1>c$ or $a+b+1 \leq c$.

Proof. The assertions $f(0)=g(0)=1$ and that $f$ is increasing, so that if $a+b<c$, then $g$ is increasing, are all clear. In the three cases, $a+b<c, a+b=c$ and $a+b>c$, the limiting values at $\infty$ are clear by (3.9). Next, by (3.9), $g(x)=F\left(c-a, c-b ; c ; 1-e^{-x}\right)$, which is clearly increasing. Next, by differentiation,

$$
c /(a b) f^{\prime}(x)=e^{-x} F\left(a+1, b+1 ; c+1 ; 1-e^{-x}\right) .
$$

Hence, by (3.9)

$$
\begin{aligned}
c /(a b) f^{\prime}(x) & =\left(e^{-x}\right)\left(e^{-x(c-a-b-1)}\right) F\left(c-a, c-b ; c+1 ; 1-e^{-x}\right) \\
& =e^{x(a+b-c)} F\left(c-a, c-b ; c+1 ; 1-e^{-x}\right),
\end{aligned}
$$

so that $h(x)=(a b / c) F\left(c-a, c-b ; c+1 ; 1-e^{-x}\right)$, which is increasing, with boundary values $h(0)=a b / c$, and by (3.9) and (3.2),

$$
h(\infty)=(a b / c) \frac{\Gamma(c+1) \Gamma(a+b+1-c)}{\Gamma(a+1) \Gamma(b+1)}=\frac{\Gamma(c) \Gamma(a+b+1-c)}{\Gamma(a) \Gamma(b)},
$$

if $a+b+1>c$, and $=\infty$ if $a+b+1 \leq c$. 
3.15. Theorem. For $a, b, c, d>0$, with $a+b>c>\max \{a, b\}$, let the function $f$ be defined on $[0, \infty)$ by $f(x)=F\left(a, b ; c ; 1-(1+x)^{-1 / d}\right)$, and let

$$
g(x)=(1+x)^{((c+d)-(a+b)) / d} f^{\prime}(x) .
$$

Then $g$ is increasing, with

$$
g(0)=\frac{a b}{c d} \text { and } g(\infty)=\frac{(a+b-c) \Gamma(c) \Gamma(a+b-c)}{d \Gamma(a) \Gamma(b)} .
$$

Proof. With $u=1-(1+x)^{-1 / d}$, by (3.6) and (3.9),

$$
\begin{aligned}
f^{\prime}(x) & =\frac{a b}{c d}(1+x)^{-1-(1 / d)} F(a+1, b+1 ; c+1 ; u) \\
& =\frac{a b}{c d}(1+x)^{(1 / d)(a+b-c-d)} F(c-a, c-b ; c+1 ; u),
\end{aligned}
$$

so that $g(x)=(a b /(c d)) F(c-a, c-b ; c+1 ; u)$, which is clearly positive and increasing. The boundary value $g(0)=a b /(c d)$ is clear, while the value of $g(\infty)$ follows from (3.9).

3.16. Remark. (1) With $c=a+b$, Theorem 3.14 reduces to [AQVV, Theorem 3.9]. (2) For $a+b=c+d$, Theorem 3.15 reduces to AQVV, Theorem 3.10.].
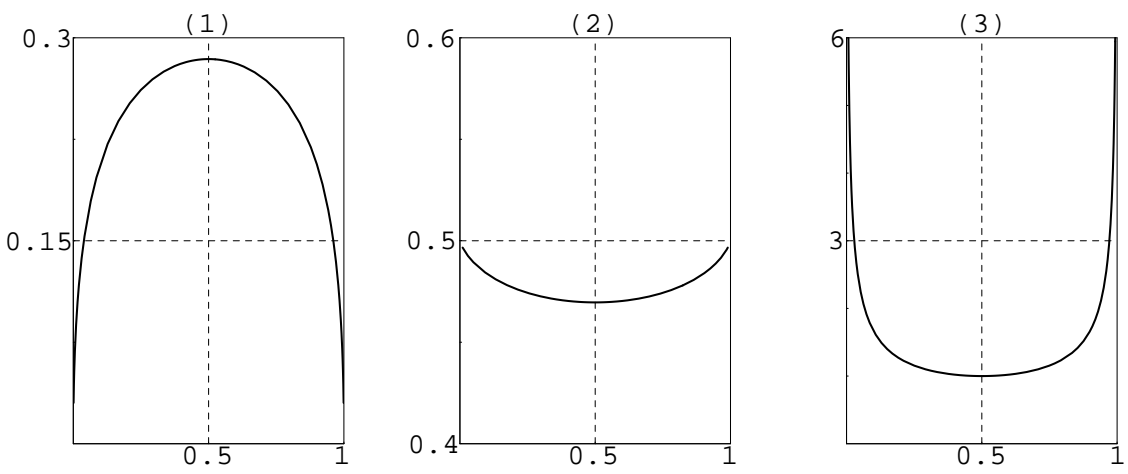

Figure 3: (1) $\mathcal{M}(0.5,1.0,2.0, \cdot),(2) \mathcal{M}(0.5,1.0,1.5, \cdot),(3) \mathcal{M}(0.5,1.0,1.0, \cdot)$

3.17. Theorem. For positive constants $a, b, c$, the restriction to $(0,1)$ of the continuous function $\mathcal{M}$ in (3.10) has the following properties. Denote $\mathcal{M}(x)=\mathcal{M}(a, b, c, x)$.

(1) $\mathcal{M}(x)=\mathcal{M}(1-x)>0$ for all $x \in(0,1)$.

(2) If $a+b \leq c$, then $\mathcal{M}(x)$ is bounded and extends continuously to $[0,1]$. In particular, if $a+b=c=1$, then $\mathcal{M}(x)$ equals the constant $\sin (\pi a) / \pi$. 
(3) If $a+b>c$, then $\mathcal{M}$ is unbounded on $(0,1)$, with $\mathcal{M}(0+)=\mathcal{M}(1-)=\infty$.

(4) If $a+b<c<a+b+1$, then

$$
\lim _{x \rightarrow 0+} x^{a+b-c} \mathcal{M}(x)=\lim _{x \rightarrow 1-}(1-x)^{a+b-c} \mathcal{M}(x)=\frac{\Gamma(c) \Gamma(a+b+1-c)}{\Gamma(a) \Gamma(b)} .
$$

(5) If $a+b+1=c$, then

$$
\lim _{x \rightarrow 0+} \frac{\mathcal{M}(x)}{x \log (1 / x)}=\lim _{x \rightarrow 1-} \frac{\mathcal{M}(x)}{(1-x) \log (1 /(1-x))}=\frac{a+b}{B(a, b)} .
$$

(6) If $a+b+1<c$, then

$$
\lim _{x \rightarrow 0+} \frac{\mathcal{M}(x)}{x}=\lim _{x \rightarrow 1-} \frac{\mathcal{M}(x)}{1-x}=\frac{a b(2 c-a-b-1) B(c, c-a-b)}{c(c-a-b-1) B(c-a, c-b)} .
$$

(7) If $a+b>c$, then

$$
\lim _{x \rightarrow 0+} x^{a+b-c} \mathcal{M}(x)=\lim _{x \rightarrow 1-}(1-x)^{a+b-c} \mathcal{M}(x)=\frac{(a+b-c) B(c, a+b-c)}{B(a, b)} .
$$

Proof. (1) From (3.8) and (3.10), we get

$$
\begin{aligned}
\mathcal{M}(x)= & \frac{a b x(1-x)}{c}(F(a+1, b+1 ; c+1 ; x) v(1-x) \\
& +F(a+1, b+1 ; c+1 ; 1-x) v(x)) \\
= & G(x)+H(x)>0 .
\end{aligned}
$$

Here

$$
G(x)=\frac{a b x(1-x)}{c} F(a+1, b+1 ; c+1 ; x) v(1-x)
$$

and

$$
H(x)=\frac{a b x(1-x)}{c} F(a+1, b+1 ; c+1 ; 1-x) v(x)=G(1-x) .
$$

(2) First, if $a+b<c$, then from (3.9) and (3.10),

$$
\mathcal{M}(0+)=\mathcal{M}(1-)=(c-a) u(1)+(a+b-c) v(1)=0 .
$$

Next, if $a+b=c$, then from (3.9) $), G(0+)=0$ and

$$
H(x)=\frac{a b(1-x)}{c} F(a, b ; a+b+1 ; 1-x) v(x),
$$

so that $H(0+)=(a b / c) F(a, b ; a+b+1 ; 1)$. Next, $H(1-)=0$ and

$$
G(x)=\frac{a b x}{c} F(a, b ; a+b+1 ; x) v(1-x),
$$

so that $G(1-)=(a b / c) F(a, b ; a+b+1 ; 1)$. Thus,

$$
\mathcal{M}(0+)=\mathcal{M}(1-)=(a b / c) F(a, b ; a+b+1 ; 1)=1 / B(a, b) .
$$


(3) Let $a+b>c$. Then

$$
H(x)=\frac{a b}{c}(1-x) x^{c-a-b} F(c-a, c-b ; c+1 ; 1-x) v(x),
$$

so that $H(0+)=\infty$. Similarly, $G(1-)=\infty$.

(4) By (3.9),

$$
\begin{aligned}
\frac{c}{a b} \mathcal{M}(x)= & x(1-x) F(a+1, b+1 ; c+1 ; x) F(a, b ; c ; 1-x ;) \\
& +x^{c-a-b}(1-x) F(c-a, c-b ; c+1 ; 1-x) F(a, b ; c ; x),
\end{aligned}
$$

so that the result follows by (1).

(5) By (3.9),

$$
\begin{aligned}
\frac{c \mathcal{M}(x)}{a b x \log (1 / x)}= & x(1-x) F(a+1, b+1 ; c+1 ; x) F(a, b ; c ; 1-x) \\
& +\frac{1-x}{\log (1 / x)} F(a+1, b+1 ; c+1 ; 1-x) F(a, b ; c ; x),
\end{aligned}
$$

so that the result follows from (1) and (3.9).

(6) By (3.9),

$$
\begin{aligned}
\frac{c \mathcal{M}(x)}{a b x}= & (1-x) F(a+1, b+1 ; c+1 ; x) F(a, b ; c ; 1-x) \\
& +(1-x) F(a+1, b+1 ; c+1 ; x),
\end{aligned}
$$

so that by (1) and (3.9),

$$
\begin{aligned}
\lim _{x \rightarrow 0+} \frac{\mathcal{M}(x)}{x} & =\lim _{x \rightarrow 1-} \mathcal{M}(x) /(1-x) \\
& =\frac{a b}{c}\left(\frac{B(c, c-a-b)}{B(c-a, c-b)}+\frac{B(c+1, c-a-b-1)}{B(c-a, c-b)}\right) \\
& =\frac{a b(2 c-a-b-1)}{(c(c-a-b-1)} \frac{B(c, c-a-b)}{B(c-a, c-b)} .
\end{aligned}
$$

(7) By (3.9),

$$
\begin{aligned}
\frac{c}{a b} x^{a+b-c} \mathcal{M}(x)= & x(1-x) F(a+1, b+1 ; c+1 ; x) F(a, b ; c ; 1-x) \\
& +(1-x) F(c-a, c-b ; c+1 ; 1-x) F(a, b ; c ; x),
\end{aligned}
$$

so that by (1) and (3.9),

$$
\begin{aligned}
\lim _{x \rightarrow 0+} x^{a+b-c} \mathcal{M}(x) & =\lim _{x \rightarrow 1-}(1-x)^{a+b-c} \mathcal{M}(x) \\
& =\frac{a b}{c} F(c-a, c-b ; c+1 ; 1) \\
& =\frac{a b}{c} \frac{B(c+1, a+b+1-c)}{B(a+1, b+1)} \\
& =\frac{(a+b-c) B(c, a+b-c)}{B(a, b)} .
\end{aligned}
$$


3.18. Lemma. The function $\mathcal{M}$ in (3.10) satisfies the differentiation formula

$$
\begin{aligned}
\frac{d \mathcal{M}(a, b, c, z)}{d z}= & \frac{1}{z(1-z)}\left(( c - a ) \left[(1-c+(a+b-1) z) u(z) v_{1}(z)\right.\right. \\
& \left.+(-a-b+c+(a+b-1) z) u_{1}(z) v(z)\right] \\
& \left.+(1-2 z)\left[(c-a)(a+2 b-1)-b^{2}\right] v(z) v_{1}(z)\right)
\end{aligned}
$$

Proof. Denote $D=d / d z$. Then, by (3.6), (3.7), and the chain rule, we get

$$
\begin{gathered}
z D u=(a-1)(v-u), z(1-z) D v=(c-a) u+(a-c+b z) v \\
(1-z) D u_{1}=(1-a)\left(v_{1}-u_{1}\right), z(1-z) D v_{1}=-\left((c-a) u_{1}+(a-c+b(1-z)) v_{1}\right) .
\end{gathered}
$$

Hence, by the product rule, after simplification, we get

$$
\begin{gathered}
z(1-z) D\left(u v_{1}\right)=-(c-a) u u_{1}+(a-1)(1-z) v v_{1}+((1-a)(1-z)+(c-a-b(1-z))) u v_{1}, \\
z(1-z) D\left(u_{1} v\right)=(c-a) u u_{1}+(1-a) z v v_{1}+(a-c+(a+b-1) z) u_{1} v
\end{gathered}
$$

and

$$
z(1-z) D\left(v v_{1}\right)=(c-a)\left(u v_{1}-u_{1} v\right)+b(2 z-1) v v_{1} .
$$

Substituting these we get

$$
\begin{aligned}
z(1-z) D \mathcal{M}(a, b, c, z)= & (c-a)\left[(1-c+(a+b-1) z) u v_{1}\right. \\
& \left.+(-a-b+c+(a+b-1) z) u_{1} v\right] \\
& +(1-2 z)\left[(c-a)(a+2 b-1)-b^{2}\right] v v_{1}
\end{aligned}
$$

3.19. Remark. If we put $c=a+b=1$ in Lemma 3.18 above, then we get the familiar fact that

$$
\frac{d}{d z} \mathcal{M}(a, 1-a, 1, z)=0 .
$$

We also observe that $\operatorname{DM}(a, b, c, 1 / 2)=0$.

\section{Generalized elliptic integrals}

The following two important Theorems, 4.1 and 4.3, are indispensable in simplified proofs for monotonicity of the quotient of two functions. The first one, called L'Hôpital's Monotone Rule, appears in [AVV, Theorem 1.25], while a more general version of the second one appears in [BK] and [PV, Lemma 2.1]. 
4.1. Theorem. Let $-\infty<a<b<\infty$, and let $f, g:[a, b] \rightarrow \mathbb{R}$ be continuous on $[a, b]$, differentiable on $(a, b)$. Let $g^{\prime}(x) \neq 0$ on $(a, b)$. If $f^{\prime}(x) / g^{\prime}(x)$ is increasing (decreasing) on $(a, b)$, then so are

$$
\frac{f(x)-f(a)}{g(x)-g(a)} \text { and } \quad \frac{f(x)-f(b)}{g(x)-g(b)}
$$

If $f^{\prime}(x) / g^{\prime}(x)$ is strictly monotone, then the monotoneity in the conclusion is also strict.

4.2. Lemma. Let $\left\{a_{n}\right\}$ and $\left\{b_{n}\right\}$ be real sequences with $b_{n}>0$ for all $n$. If the sequence $\left\{a_{n} / b_{n}\right\}$ is increasing (decreasing), then

$$
T_{n}=\sum_{k=0}^{n}(n-k)\left(a_{n-k} b_{k}-a_{k} b_{n-k}\right)
$$

is positive (negative) for $n=1,2, \ldots$

Proof. It is enough to prove the case $\left\{a_{n} / b_{n}\right\}$ is increasing, since the other one is similar. Clearly

$$
T_{1}=a_{1} b_{0}-a_{0} b_{1}=b_{0} b_{1}\left(\frac{a_{1}}{b_{1}}-\frac{a_{0}}{b_{0}}\right)>0 .
$$

Let $n \geq 2$. First let $n=2 m$ be even. Then

$$
\begin{aligned}
T_{n}=T_{2 m}= & \sum_{k=0}^{2 m}(2 m-k)\left(a_{2 m-k} b_{k}-a_{k} b_{2 m-k}\right) \\
= & \sum_{k=0}^{2 m-1}(2 m-k)\left(a_{2 m-k} b_{k}-a_{k} b_{2 m-k}\right) \\
= & \sum_{k=0}^{m-1}(2 m-k)\left(a_{2 m-k} b_{k}-a_{k} b_{2 m-k}\right)+0 \\
& +\sum_{k=m+1}^{2 m-1}(2 m-k)\left(a_{2 m-k} b_{k}-a_{k} b_{2 m-k}\right) \\
= & \sum_{k=0}^{m-1}(2 m-k)\left(a_{2 m-k} b_{k}-a_{k} b_{2 m-k}\right) \\
& +\sum_{k=1}^{m-1} k\left(a_{k} b_{2 m-k}-a_{2 m-k} b_{k}\right) \\
= & 2 m\left(a_{2 m} b_{0}-a_{0} b_{2 m}\right)+\sum_{k=1}^{m-1}(2 m-2 k)\left(a_{2 m-k} b_{k}-a_{k} b_{2 m-k}\right) \\
= & 2 m b_{0} b_{2 m}\left(\frac{a_{2 m}}{b_{2 m}}-\frac{a_{0}}{b_{0}}\right)+\sum_{k=1}^{m-1}(2 m-2 k) b_{k} b_{2 m-k}\left(\frac{a_{2 m-k}}{b_{2 m-k}}-\frac{a_{k}}{b_{k}}\right)>0 .
\end{aligned}
$$


Next, let $n=2 m+1$ be odd. Then

$$
\begin{aligned}
T_{n}= & \sum_{k=0}^{2 m+1}(2 m+1-k)\left(a_{2 m+1-k} b_{k}-a_{k} b_{2 m+1-k}\right) \\
= & \sum_{k=0}^{2 m}(2 m+1-k)\left(a_{2 m+1-k} b_{k}-a_{k} b_{2 m+1-k}\right) \\
= & (2 m+1)\left(a_{2 m+1} b_{0}-a_{0} b_{2 m+1}\right)+\sum_{k=1}^{m}(2 m+1-k)\left(a_{2 m+1-k} b_{k}-a_{k} b_{2 m+1-k}\right) \\
& +\sum_{k=m+1}^{2 m}(2 m+1-k)\left(a_{2 m+1-k} b_{k}-a_{k} b_{2 m+1-k}\right) \\
= & (2 m+1)\left(a_{2 m+1} b_{0}-a_{0} b_{2 m+1}\right)+\sum_{k=1}^{m}(2 m+1-k)\left(a_{2 m+1-k} b_{k}-a_{k} b_{2 m+1-k}\right) \\
& +\sum_{k=1}^{m} k\left(a_{k} b_{2 m+1-k}-a_{2 m+1-k} b_{k}\right) \\
= & (2 m+1)\left(a_{2 m+1} b_{0}-a_{0} b_{2 m+1}\right)+\sum_{k=1}^{m}(2 m+1-2 k)\left(a_{2 m+1-k} b_{k}-a_{k} b_{2 m+1-k}\right) \\
= & (2 m+1)\left(b_{0} b_{2 m+1}\right)\left(\frac{a_{2 m+1}}{b_{2 m+1}}-\frac{a_{0}}{b_{0}}\right)^{m}\left(\frac{a_{2 m+1-k}}{b_{2 m+1-k}}-\frac{a_{k}}{b_{k}}\right)>0 . \\
& +\sum_{k=1}^{m}(2 m+1-2 k) b_{k} b_{2 m+1-k}
\end{aligned}
$$

4.3. Theorem. Let $\sum_{n=0}^{\infty} a_{n} x^{n}$ and $\sum_{n=0}^{\infty} b_{n} x^{n}$ be two real power series converging on the interval $(-R, R)$. If the sequence $\left\{a_{n} / b_{n}\right\}$ is increasing (decreasing), and $b_{n}>0$ for all $n$, then the function

$$
f(x)=\frac{\sum_{n=0}^{\infty} a_{n} x^{n}}{\sum_{n=0}^{\infty} b_{n} x^{n}}
$$

is also increasing (decreasing) on $(0, R)$. In fact, the function $f^{\prime}(x)\left(\sum_{n=0}^{\infty} b_{n} x^{n}\right)^{2}$ has positive Maclaurin coefficients.

\section{Proof.}

$$
\begin{aligned}
f^{\prime}(x)\left(\sum_{n=0}^{\infty} b_{n} x^{n}\right)^{2} & =\sum_{n=0}^{\infty} b_{n} x^{n} \sum_{n=0}^{\infty} n a_{n} x^{n-1}-\sum_{n=0}^{\infty} a_{n} x^{n} \sum_{n=0}^{\infty} n b_{n} x^{n-1} \\
& =(1 / x) \sum_{n=0}^{\infty}\left(\sum_{k=0}^{n}(n-k)\left(a_{n-k} b_{k}-a_{k} b_{n-k}\right)\right) x^{n}
\end{aligned}
$$

The result follows from Lemma 4.2.

The following theorem solves the corresponding problem in the case where we have a quotient of two polynomials instead of two power series. 
4.4. Theorem. Let $f_{n}(x)=\sum_{k=0}^{n} a_{k} x^{k}$ and $g_{n}(x)=\sum_{k=0}^{n} b_{k} x^{k}$ be real polynomials, with $b_{k}>0$ for all $k$. If the sequence $\left\{a_{k} / b_{k}\right\}$ is increasing (decreasing), then so is the function $f_{n}(x) / g_{n}(x)$ for all $x>0$. In fact, $g_{n} f_{n}^{\prime}-f_{n} g_{n}^{\prime}$ has positive (negative) coefficients.

Proof. We prove the increasing case by induction on $n$. The proof of the decreasing case is similar. Let first $n=1$. Then

$$
\frac{f_{1}(x)}{g_{1}(x)}=\frac{a_{0}+a_{1} x}{b_{0}+b_{1} x} .
$$

Hence

$$
\begin{aligned}
g_{1} f_{1}^{\prime}-f_{1} g_{1}^{\prime} & =\left(b_{0}+b_{1} x\right) a_{1}-\left(a_{0}+a_{1} x\right) b_{1} \\
& =a_{1} b_{0}-a_{0} b_{1} \\
& =b_{0} b_{1}\left(\frac{a_{1}}{b_{1}}-\frac{a_{0}}{b_{0}}\right)>0 .
\end{aligned}
$$

Next, assume that the claim holds for all $k \leq n$. Now

$$
\frac{f_{n+1}(x)}{g_{n+1}(x)}=\frac{f_{n}(x)+a_{n+1} x^{n+1}}{g_{n}(x)+b_{n+1} x^{n+1}} .
$$

We get

$$
\begin{aligned}
g_{n+1} f_{n+1}^{\prime}-f_{n+1} g_{n+1}^{\prime}= & \left(g_{n}+b_{n+1} x^{n+1}\right)\left(f_{n}^{\prime}+(n+1) a_{n+1} x^{n}\right) \\
& -\left(f_{n}+a_{n+1} x^{n+1}\right)\left(g_{n}^{\prime}+(n+1) b_{n+1} x^{n}\right) \\
= & \left(g_{n} f_{n}^{\prime}-f_{n} g_{n}^{\prime}\right)+(n+1) x^{n}\left(g_{n} a_{n+1}-f_{n} b_{n+1}\right) \\
& +x^{n+1}\left(b_{n+1} f_{n}^{\prime}-a_{n+1} g_{n}^{\prime}\right) \\
= & \left(g_{n} f_{n}^{\prime}-f_{n} g_{n}^{\prime}\right)+(n+1) x^{n} \sum_{k=0}^{n}\left(a_{n+1} b_{k}-b_{n+1} a_{k}\right) x^{k} \\
& +x^{n+1} \sum_{k=1}^{n} k\left(a_{k} b_{n+1}-b_{k} a_{n+1}\right) x^{k-1} \\
= & \left(g_{n} f_{n}^{\prime}-f_{n} g_{n}^{\prime}\right)+\sum_{k=0}^{n}(n+1-k)\left(a_{n+1} b_{k}-b_{n+1} a_{k}\right) x^{n+k} \\
= & \left(g_{n} f_{n}^{\prime}-f_{n} g_{n}^{\prime}\right)+\sum_{k=0}^{n}(n+1-k) b_{k} b_{n+1}\left(\frac{a_{n+1}}{b_{n+1}}-\frac{a_{k}}{b_{k}}\right) x^{n+k} .
\end{aligned}
$$

Hence each coefficient is positive.

4.5. Lemma. Let $a, b, c, K>0$.

(1) If $a+b \geq c$, then $\mu_{a, b, c}:(0,1) \rightarrow(0, \infty)$ and $\varphi_{K}^{a, b, c}:(0,1) \rightarrow(0,1)$ are decreasing and increasing homeomorphisms, respectively.

(2) If $a+b>c$, then the function $f(r)=\left(r / r^{\prime}\right)^{2(a+b-c)} \mu_{a, b, c}(r)$ is decreasing from $(0,1)$ onto $\left(\left(B(a, b)^{2}\right) /(2 B(c, a+b-c)), B(c, a+b-c) / 2\right)$. 
(3) If $a+b>c$ and $K>1$, then

$$
r<\varphi_{K}^{a, b, c}(r)<K^{1 /(2(a+b-c))} r
$$

for all $r \in(0,1)$.

(4) If $a+b<c$, then $\mu_{a, b, c}:[0,1] \rightarrow[B(a, b) /(2 d), B(a, b) d / 2]$, is a decreasing homeomorphism, where $d=F(a, b ; c ; 1)$ is given by (3.9). In this case, $\varphi_{K}^{a, b, c}$ is defined if and only if $K \geq 1$. If $K>1$, then $\varphi_{K}^{a, b, c}$ maps $[0,1]$ onto a proper subset of $[0,1]$.

Proof. (1) Since $a, b, c>0$ and $a+b \geq c$, it follows from (3.9) that the function $F(a, b ; c ; r)$ is an increasing homeomorphism of $[0,1)$ onto $[1, \infty)$. Hence the function $\mu_{a, b, c}$ is a strictly decreasing homeomorphism $(0,1) \rightarrow(0, \infty)$. The assertion about $\varphi_{K}^{a, b, c}$ follows from these facts.

(2) From (3.9),

$$
f(r)=\frac{B(a, b)}{2} \frac{F\left(c-a, c-b ; c ; r^{2}\right)}{F\left(c-a, c-b ; c ; r^{2}\right)},,
$$

which is decreasing with required limiting values at 0 and 1.

(3) With $s=\varphi_{K}^{a, b, c}(r)>r$, from (2) we get

$$
\begin{aligned}
f(s) & =\left(s / s^{\prime}\right)^{2(a+b-c)} \mu_{a, b, c}(s)=\left(s / s^{\prime}\right)^{2(a+b-c)} \mu_{a, b, c}(r) / K \\
& <f(r)=\left(r / r^{\prime}\right)^{2(a+b-c)} \mu_{a, b, c}(r)
\end{aligned}
$$

so that

$$
\left(s / s^{\prime}\right)^{2(a+b-c)}<K\left(r / r^{\prime}\right)^{2(a+b-c)},
$$

which gives $s / s^{\prime}<K^{1 /(2(a+b-c))} r / r^{\prime}$. Hence

$$
r<s<K^{1 /(2(a+b-c))} r\left(s^{\prime} / r^{\prime}\right)<K^{1 /(2(a+b-c))} r .
$$

(4) For $a, b, c>0$ and $a+b<c$, (3.9) implies that $F(a, b ; c ; r)$ is an increasing homeomorphism $[0,1] \rightarrow[1, F(a, b ; c ; 1)]$. Now the claim follows from (1.3) and (1.5).

The function $\mu_{a, b, c}$ is a natural generalization for the function $\mu_{a}$ in AQVV, (1.3)]. Namely,

$$
\mu_{a, 1-a, 1}(r)=\frac{B(a, 1-a)}{2} \frac{F\left(a, 1-a ; 1 ; r^{\prime 2}\right)}{F\left(a, 1-a ; 1 ; r^{2}\right)}=\mu_{a}(r)
$$

since by (3.4) and (3.5),

$$
B(a, 1-a)=\frac{\Gamma(a) \Gamma(1-a)}{\Gamma(1)}=\frac{\pi}{\sin (\pi a)} .
$$

Clearly (1.3) and (1.5) imply the identities (cf. AQVV, (4.8), (4.9)]),

$$
\mu_{a, b, c}(r) \mu_{a, b, c}\left(r^{\prime}\right)=\left(\frac{B(a, b)}{2}\right)^{2}, r \in(0,1),
$$



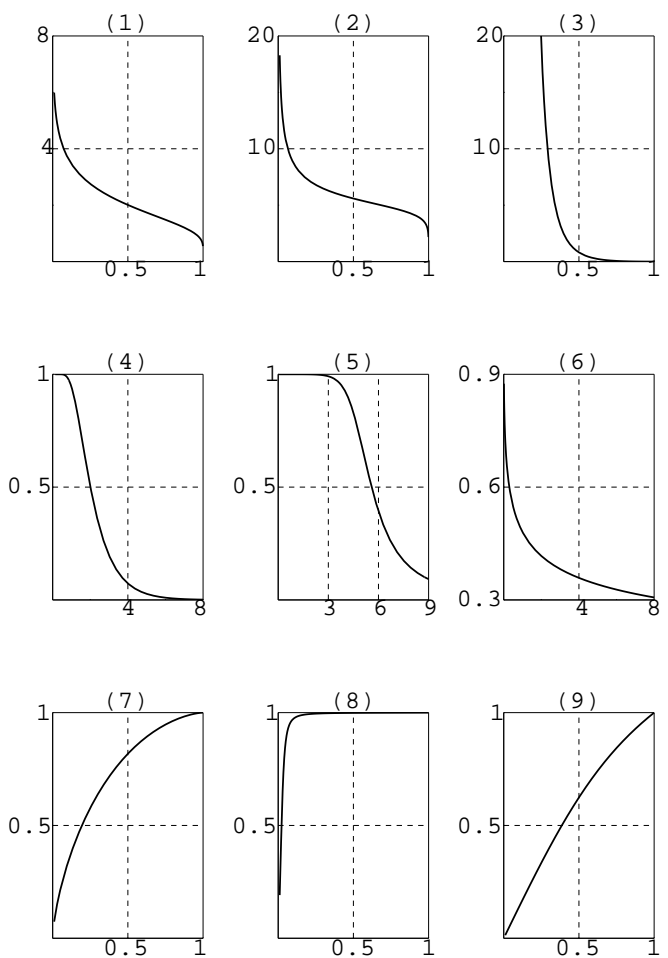

Figure 4: (1) $\mu_{0.5,0.5,1}$, (2) $\mu_{0.1,1.1,1}$, (3) $\mu_{2.5,1.5,2}$, (4) $\mu_{0.5,0.5,1}^{-1}$, (5) $\mu_{0.1,1.1,1}^{-1}$, (6) $\mu_{2.5,1.5,2}^{-1}$, (7) $\varphi_{1.5}^{0.5,0.5,1}$, (8) $\varphi_{2.5}^{0.1,1.1,1}$, (9) $\varphi_{3.5}^{2.5,1.5,2}$.

$$
\mu_{a, b, c}^{-1}(x)^{2}+\mu_{a, b, c}^{-1}(y)^{2}=1
$$

where $x, y>0$ with $x y=(B(a, b) / 2)^{2}$, and

$$
\varphi_{K}^{a, b, c}(r)^{2}+\varphi_{1 / K}^{a, b, c}\left(r^{\prime}\right)^{2}=1 .
$$

Moreover, from (1.3) and (3.9) we get, for $c<a+b$,

$$
r^{\prime 2(c-a-b)} \mu_{a, b, c}(r)=r^{2(c-a-b)} \mu_{c-a, c-b, c}(r), \quad r \in(0,1) .
$$

4.11. Lemma. Let $f$ be a bijection from a real interval $I$ onto $(0, \infty)$ and let $g$ be defined on $I$ by $g(x)=a f(x)$, where $a>0$ is a constant. Then for each constant $K>0$, we have

$$
f^{-1}(K f(x))=g^{-1}(K g(x)) .
$$

Proof. Let $u=f^{-1}(K f(x))$. Then $f(u)=K f(x)$, so that $a f(u)=a K f(x)$, that is, $g(u)=K g(x)$. Hence $u=g^{-1}(K g(x))$. 
4.12. Remark. For $a, b, c>0$ with $a+b \geq c, K>0$, and $r \in(0,1)$ denote

$$
\tilde{\mu}_{a, b, c}(r)=\frac{F\left(a, b ; c ; r^{2}\right)}{F\left(a, b ; c ; r^{2}\right)}
$$

and

$$
\tilde{\varphi}_{K}^{a, b, c}(r)=\tilde{\mu}_{a, b, c}^{-1}\left(\tilde{\mu}_{a, b, c}(r) / K\right) .
$$

By Lemma 4.11 we see that

$$
\tilde{\varphi}_{K}^{a, b, c}(r)=\varphi_{K}^{a, b, c}(r)
$$

4.13. Remark. Observe first that $\lim _{c \rightarrow a+} B(a, c-a)=\infty$ and $\Gamma(c-a)(c-a, n)=$ $\Gamma(c-a+n)$, which tends to $\Gamma(n)=(n-1)$ !, as $c \rightarrow a+$.

(1) $\lim _{c \rightarrow a+}\left(\mathcal{K}_{a, c}(r)-(B(a, c-a) / 2)\right)=\log \left(1 / r^{\prime}\right)$.

(2) $\lim _{c \rightarrow a+}\left(\mathcal{E}_{a, c}(r)-(B(a, c-a) / 2)\right)=\log \left(1 / r^{\prime}\right)-\left(\frac{1}{2} \sum_{n=1}^{\infty} \frac{r^{2 n}}{a+n-1}\right)$.

In particular, for each fixed $r \in(0,1)$ all the three functions $\mathcal{K}_{a, c}(r), \mathcal{E}_{a, c}(r)$, and $\mathcal{E}_{a, c}(r)-r^{\prime 2} \mathcal{K}_{a, c}(r)$ tend to $\infty$ as $c \rightarrow a+$.

4.14. Theorem. The following differentiation formulae hold:

$$
\begin{gathered}
\frac{d \mathcal{K}_{a, b, c}}{d r}=\frac{2}{r r^{\prime 2}}\left((c-a) \mathcal{E}_{a, b, c}+\left(b r^{2}+a-c\right) \mathcal{K}_{a, b, c}\right) \\
\frac{d \mathcal{E}_{a, b, c}}{d r}=\frac{2(a-1)}{r}\left(\mathcal{K}_{a, b, c}-\mathcal{E}_{a, b, c}\right) \\
\frac{d}{d r}\left(\mathcal{K}_{a, b, c}-\mathcal{E}_{a, b, c}\right)=\frac{2}{r r^{\prime 2}}\left(\left((c-a)-(1-a) r^{\prime 2}\right) \mathcal{E}_{a, b, c}\right. \\
\left.+\left((a+b) r^{2}-c+r^{\prime 2}\right) \mathcal{K}_{a, b, c}\right) \\
\frac{d}{d r}\left(\mathcal{E}_{a, b, c}-r^{\prime 2} \mathcal{K}_{a, b, c}\right)=\frac{2}{r}\left((1-c) \mathcal{E}_{a, b, c}+\left(c-1-(b-1) r^{2}\right) \mathcal{K}_{a, b, c}\right), \\
\frac{d}{d r} \mu_{a, b, c}(r)=-\frac{B(a, b) \mathcal{M}\left(a, b, c, r^{2}\right)}{r r^{\prime 2} v\left(r^{2}\right)^{2}}=-\frac{B(a, b)^{3} \mathcal{M}\left(r^{2}\right)}{4 r r^{\prime 2} \mathcal{K}^{2}} \\
\frac{\mathcal{M}\left(a, b, c, s^{2}\right)}{\mathcal{M}\left(a, b, c, r^{2}\right)} \frac{d s}{d r}=\frac{1}{K} \frac{s s^{\prime 2} v\left(s^{2}\right)^{2}}{r r^{\prime 2} v\left(r^{2}\right)^{2}}=\frac{1}{K} \frac{s s^{\prime 2} \mathcal{K}(s)^{2}}{r r^{\prime 2} \mathcal{K}(r)^{2}}, s=\varphi_{K}^{a, b, c}(r) .
\end{gathered}
$$

Proof. From (3.6)

$$
\frac{d F}{d z}=\frac{(c-a) u+(a-c+b z) v}{z(1-z)} .
$$

Put $z=r^{2}$ and multiply both sides by $B(a, b) / 2$. This gives (4.15). 
For (4.16) recall that by (3.7)

$$
\frac{d u}{d z}=\frac{(a-1)}{z}(v-u)
$$

Put $z=r^{2}$ and multiply both sides by $B(a, b) / 2$. This gives (4.16).

The formulae (4.17) and (4.18) follow from (4.15) and (4.16).

From (3.10), putting $z=r^{2}$, we get

$$
\mathcal{M}\left(r^{2}\right)=-r^{2} r^{\prime 2} \frac{d \mu}{d r}\left(\frac{2}{B(a, b)}\right)^{3} \mathcal{K}^{2}\left(\frac{1}{2 r}\right),
$$

so that

$$
\frac{d \mu}{d r}=-\frac{B(a, b)^{3} \mathcal{M}\left(r^{2}\right)}{4 r r^{\prime 2} \mathcal{K}^{2}} .
$$

Denote $s=\varphi_{K}^{a, b, c}(r)$. Then by (1.5)

$$
\mu_{a, b, c}(s)=\frac{1}{K} \mu_{a, b, c}(r)
$$

so that

$$
\frac{d}{d s} \mu_{a, b, c}(s) \frac{d s}{d r}=\frac{1}{K} \frac{d}{d r} \mu_{a, b, c}(r) .
$$

Now (4.20) follows from (4.19).

4.21. Lemma. (cf. $A Q V V$, Lemmas 5.2 and 5.4]) For $0<a, b<\min \{c, 1\}$ and $c \leq a+b$, denote $\mathcal{K}=\mathcal{K}_{a, b, c}$ and $\mathcal{E}=\mathcal{E}_{a, b, c}$. Then the function

(1) $f_{1}(r)=(\mathcal{K}-\mathcal{E}) /\left(r^{2} \mathcal{K}\right)$ is strictly increasing from $(0,1)$ onto $(b / c, 1)$. In particular, we have the sharp inequality,

$$
\frac{b}{c}<\frac{\mathcal{K}-\mathcal{E}}{r^{2} \mathcal{K}}<1
$$

for all $r \in(0,1)$.

(2) $f_{2}(r)=\left(\mathcal{E}-r^{\prime 2} \mathcal{K}\right) / r^{2}$ has positive Maclaurin coefficients and maps $(0,1)$ onto $(B(a, b)(c-b) /(2 c), C)$, where

$$
C=\frac{B(a, b) B(c, c+1-a-b)}{2 B(c+1-a, c-b)} .
$$

(3) $f_{3}(r)=r^{-2(c+1-a-b)} \mathcal{E}$ has positive Maclaurin coefficients and maps $[0,1)$ onto $(B(a, b) / 2, \infty)$.

(4) $f_{4}(r)=r^{2(a+b-c)} \mathcal{K}$ has positive Maclaurin coefficients and maps $[0,1)$ onto $[B(a, b) / 2, B(c, a+b-c) / 2)$.

(5) $f_{5}(r)=r^{-2} \mathcal{E}$ has positive Maclaurin coefficients and maps $[0,1)$ onto $[B(a, b) / 2, \infty)$.

(6) $f_{6}(r)=r^{\prime 2} \mathcal{K}$ has negative Maclaurin coefficients, except for the constant term, and maps $[0,1)$ onto $(0, B(a, b) / 2]$.

(7) $f_{7}(r)=\mathcal{K}$ has positive Maclaurin coefficients and is log-convex from $[0,1)$ onto $[B(a, b) / 2, \infty)$. In fact, $(d / d r)(\log \mathcal{K})$ also has positive Maclaurin coefficients. 
(8) $f_{8}(r)=\left(\mathcal{E}-r^{\prime 2} \mathcal{K}\right) /\left(r^{2} \mathcal{K}\right)$ is strictly decreasing from $(0,1)$ onto $(0,1-(b / c))$.

(9) $f_{9}(r)=(\mathcal{K}-\mathcal{E}) /\left(\mathcal{E}-{r^{\prime}}^{2} \mathcal{K}\right)$ is strictly increasing from $(0,1)$ onto $(b /(c-b), \infty)$.

(10) $f_{10}(r)=(\mathcal{K}-B(a, b) / 2) / \log \left(1 / r^{\prime}\right)$ is strictly increasing from $(0,1)$ onto $(a b B(a, b) / c, D)$, where $D=1$ if $c=a+b$ and $D=\infty$ if $c<a+b$.

(11) $f_{11}(r)=\left(B(a, b) / 2-r^{\prime 2} \mathcal{K}\right) / r^{2}$ has positive Maclaurin coefficients and maps $(0,1)$ onto $(B(a, b)(c-a b) /(2 c), B(a, b) / 2)$.

(12) $f_{12}(r)=(\mathcal{K}-B(a, b) / 2) /\left(r^{\prime 2(c-a-b)}-1\right)$, for $a+b>c$, is strictly increasing from $(0,1)$ onto $(a b B(a, b) /(2 c(a+b-c)), B(c, a+b-c) / 2)$.

(13) $f_{13}(r)=\left(\left(1-a-(b-c) r^{2}\right) \mathcal{E}-(1-a){r^{\prime}}^{2} \mathcal{K}\right) / r^{2}$ has negative Maclaurin coefficients, except for the constant term, with

$$
f_{13}(0+)=(c+1-a)(c-b) B(a, b) /(2 c)
$$

and $f_{13}(1-)=(c+1-a-b) \mathcal{E}(1)$.

(14) $f_{14}(r)=(c-a) \mathcal{E}-(b-a) r^{\prime 2} \mathcal{K}$ has negative Maclaurin coefficients, except for the constant term, and maps $[0,1]$ onto $[C, D]$, where

$$
C=(c-a) \mathcal{E}(1) \text { and } D=(c-b) B(a, b) / 2 .
$$

Proof. (1) From (3.6)

$$
\frac{d F\left(a^{-}\right)}{d z}=\frac{(a-1)}{z}\left(F-F\left(a^{-}\right)\right)=\frac{(a-1) b}{c} F(a, b+1 ; c+1 ; z) .
$$

Putting $z=r^{2}$ and multiplying by $B(a, b) / 2$, we get

$$
\frac{\mathcal{K}-\mathcal{E}}{r^{2}}=\frac{b B(a, b)}{2 c} F\left(a, b+1 ; c+1 ; r^{2}\right) .
$$

Hence

$$
f_{1}(r)=\frac{b}{c} \frac{F\left(a, b+1 ; c+1 ; r^{2}\right)}{F\left(a, b ; c ; r^{2}\right)} .
$$

Thus $f_{1}(0)=b / c$. The ratio of the coefficients of the numerator and denominator equals

$$
\frac{b}{c} \frac{(a, n)(b+1, n)(c, n)}{(c+1, n)(a, n)(b, n)}=\frac{b}{c} \frac{b+n}{b} \frac{c}{c+n}=\frac{b+n}{c+n}=1-\frac{c-b}{c+n},
$$

which is increasing in $n$. Hence the result follows from Theorem 4.3. The limit $f_{1}(1-)=1$ follows from (3.9).

(2) From (3.6) and (3.7), we get

$$
\frac{d F(a-)}{d z}=\frac{(a-1)}{z}(F-F(a-))=(a-1)\left(F+\frac{(b-c)}{c} F(c+)\right) .
$$

Hence putting $z=r^{2}$, we get

$$
\frac{\mathcal{K}-\mathcal{E}}{r^{2}}=\mathcal{K}+\frac{(b-c) B(a, b)}{2 c} F\left(a, b ; c+1 ; r^{2}\right) .
$$

Thus,

$$
f_{2}(r)=\frac{(c-b) B(a, b)}{2 c} F\left(a, b ; c+1 ; r^{2}\right),
$$


which proves the assertion. The limiting values follow from (3.9).

(3) By (3.9),

$$
\begin{aligned}
f_{3}(r) & =\frac{B(a, b)}{2} \frac{F\left(a-1, b ; c ; r^{2}\right)}{r^{2(c+1-a-b)}} \\
& =\frac{B(a, b)}{2} F\left(c+1-a, c-b ; c ; r^{2}\right),
\end{aligned}
$$

and the result follows.

(4) By (3.9),

$$
\begin{aligned}
f_{4}(r) & =\frac{B(a, b)}{2} r^{2(a+b-c)} F\left(a, b ; c ; r^{2}\right) \\
& =\frac{B(a, b)}{2} F\left(c-a, c-b ; c ; r^{2}\right),
\end{aligned}
$$

and the result follows.

(5) By (3.9)

$$
f_{5}(r)=\frac{B(a, b)}{2{r^{\prime}}^{2}} F\left(a-1, b ; c ; r^{2}\right)=\frac{B(a, b)}{2}\left(1-r^{2}\right)^{-(a+b-c)} F\left(c+1-a, c-b ; c ; r^{2}\right),
$$

which is a product of two Maclaurin series with positive coefficients, hence the result.

(6) We have that

$$
f_{6}(r)=\mathcal{E}(r)-\left(\mathcal{E}(r)-r^{\prime 2} \mathcal{K}(r)\right) .
$$

Hence, by $(2), f_{6}(r)-B(a, b) / 2$ has all Maclaurin coefficients negative.

(7) The positivity of Maclaurin coefficients and the limiting values are clear. Next, by (4.15), after simplification, we get

$$
\frac{d}{d r} \log \mathcal{K}=\frac{2 r}{r^{\prime 2} \mathcal{K}}\left((a+b-c) \mathcal{K}+\frac{(c-a)\left(\mathcal{E}-r^{\prime 2} \mathcal{K}\right)}{r^{2}}\right),
$$

which has positive Maclaurin coefficients by (2) and (6).

(8) $f_{8}(r)=1-f_{1}(r)$, so that the result follows from (1).

(9) $f_{9}(r)=f_{1}(r) / f_{8}(r)$, hence the result follows from (1) and (8).

(10) The ratio of the coefficients equals

$$
\frac{B(a, b)(a, n)(b, n)}{(c, n)(n-1) !} \equiv T_{n}
$$

say. Then

$$
\frac{T_{n+1}}{T_{n}}=\frac{(a+n)(b+n)}{(c+n) n}>1,
$$

so that the monotonicity follows from 4.3. The limiting values follow from (3.9)).

(11)

$$
\begin{aligned}
f_{11}(r) & =\mathcal{K}-\frac{\mathcal{K}-B / 2}{r^{2}} \\
& =\frac{B}{2}\left(1-\frac{a b}{c}+\sum_{n=1}^{\infty}\left(\frac{(a, n)(b, n)}{(c, n) n !}\left(1-\frac{(a+n)(b+n)}{(c+n)(n+1)}\right) r^{2 n}\right)\right),
\end{aligned}
$$


which has all coefficients positive. The limiting values are obvious.

(12) The ratio has the indeterminate form $0 / 0$ at $r=0$. The derivative ratio equals

$$
\frac{a b B(a, b)}{2 c(a+b-c)} \frac{F\left(a+1, b+1 ; c+1 ; r^{2}\right)}{r^{\prime 2(c-a-b-1)}}=\frac{a b B(a, b)}{2 c(a+b-c)} F\left(c-a, c-b ; c+1 ; r^{2}\right),
$$

by (3.9), and so the result follows by Lemma 4.1 ,

(13) From (3.6) and (3.7)

$$
\begin{aligned}
\frac{d F(a-)}{d z} & =\frac{1}{1-z}\left((a-1+b-c) F(a-)+\frac{(c+1-a)(c-b)}{c} F(a-, c+)\right) \\
& =\frac{(a-1)}{z}(F-F(a-)) .
\end{aligned}
$$

Multiplying by $z(1-z) B(a, b) / 2$, we get

$$
z(a-1+b-c) \mathcal{E}+\frac{(c+1-a)(c-b)}{c} z F(a-, c+) \frac{B(a, b)}{2}=(a-1)(1-z)(\mathcal{K}-\mathcal{E}) .
$$

With $z=r^{2}$ this yields the result.

(14) In the Gauss contiguous relation in [R, Exercise 21(9), p. 71], if we change $z$ to $r^{2}$ and multiply by $B(a, b) / 2$, then we get $f_{14}(r)=((c-b) B(a, b) / 2) F\left(a, b-1 ; c ; r^{2}\right)$, so that the assertions on the coefficients follow. The limiting values are clear.

4.22. Remark. In the classical case, $a=b=1 / 2$ and $c=1$, the boundary values in the above result 4.21 (13) reduce to $f_{13}(0+)=3 \pi / 8$ and $f_{13}(1-)=1$, showing that the above result is quite sharp.

4.23. Theorem. Let $0<a<c \leq 1, b=c-a, R=R(a, c-a)=-\Psi(a)-\Psi(c-$ a) $-2 \gamma$, and $B=B(a, c-a)$. Then

(1) The function $f(r)=\mathcal{K}_{a, c}(r)+\log r^{\prime}$ has negative Maclaurin coefficients, except for the constant term, and maps $[0,1)$ onto $(R / 2, B / 2]$.

(2) The function $g(r)=\mathcal{K}_{a, c}(r)+\left(1 / r^{2}\right) \log r^{\prime}$ has positive Maclaurin coefficients and maps $(0,1)$ onto $((B-1) / 2, R / 2)$ if $a, b \in(0,1)$, while it has negative Maclaurin coefficients and maps $(0,1)$ onto $(R / 2,(B-1) / 2)$ if $a, b \in(1, \infty)$.

(3) The function $h(r)=r^{2} \mathcal{K}_{a, c}(r) / \log \left(1 / r^{\prime}\right)$ is strictly decreasing (respectively, increasing) from $(0,1)$ onto $(1, B)$ if $a, b \in(0,1)$ (respectively, onto $(B, 1)$, if $a, b \in(1, \infty)$ ).

(4) The function $k(r)=\mathcal{K}_{a, c}(r) / \log \left(\left(e^{R / 2}\right) / r^{\prime}\right)$ is strictly decreasing from $(0,1)$ onto $(1, B / R)$.

Proof. (1) That $f(0+)=B(a, c-a) / 2$ is clear and $f(1-)=R(a, c-a) / 2$ follows from AVV, Theorem $1.52(2)]$. Next,

$$
2 f(r)=B+B \sum_{n=1}^{\infty}\left(\frac{(a, n)(b, n)}{(c, n) n !}-\frac{1}{n}\right) r^{2 n} .
$$


Thus, we need to show that $T_{n}=(a, n)(b, n) /((c, n)(n-1) !)<1$. Now

$$
\frac{T_{n+1}}{T_{n}}=\frac{(a+n)(b+n)}{(c+n) n}=\frac{(a+b) n+n^{2}+a b}{(a+b) n+n^{2}}>1
$$

and $\lim _{n \rightarrow \infty} T_{n}=1$ by Stirling's formula. Hence $T_{n}<1$ for all $n=1,2,3, \ldots$.

$(2)$

$$
g(0+)=\frac{B}{2}+\lim _{r \rightarrow 0} \frac{\log r^{\prime}}{r^{2}}=\frac{B}{2}-\frac{1}{2} .
$$

Next, $g(r)=f(r)+\left(1 / r^{2}-1\right) \log r^{\prime}$, so that $g(1-)=f(1-)=R / 2$, from (1). Next,

$$
\begin{aligned}
2 g(r) & =B F\left(a, b ; c ; r^{2}\right)-\frac{1}{r^{2}} \log \frac{1}{1-r^{2}} \\
& =\sum_{n=0}^{\infty} \frac{1}{n+1}\left(\frac{B(a, n)(b, n)(n+1)}{(c, n) n !}-1\right) r^{2 n} .
\end{aligned}
$$

Let

$$
T_{n}=\frac{B(a, n)(b, n)(n+1)}{(c, n) n !}
$$

Then

$$
\frac{T_{n+1}}{T_{n}}=\frac{(a+n)(b+n)(n+2)}{(c+n)(n+1)^{2}} .
$$

Now $(a+n)(b+n)(n+2)-(c+n)(n+1)^{2}=-(1-a b) n-(a+b-2 a b)$, which is negative (positive) if $a, b \in(0,1)(a, b \in(1, \infty))$. By Stirling's formula, $\lim _{n \rightarrow \infty} T_{n}=1$. Hence the result follows.

(3)

$$
h(r)=\frac{g(r)}{\frac{1}{r^{2}} \log \frac{1}{r^{\prime}}}+1,
$$

so that $h(0+)=B$ and $h(1-)=1$ are clear. Next,

$$
h(r)=\frac{B \sum_{n=0}^{\infty} \frac{(a, n)(b, n)}{(c, n) n !} r^{2 n}}{\sum_{n=0}^{\infty} \frac{1}{n+1} r^{2 n}},
$$

so that the coefficient ratio equals $(a, n)(b, n)(n+1) /((c, n) n !)$, which is decreasing if $a, b \in(0,1)$ and increasing if $a, b \in(1, \infty)$. Hence the result follows from [PV] Lemma $2.1]$.

(4) We have $k(r)=1+(f(r)-R / 2) / \log \left(e^{R / 2} / r^{\prime}\right)$. Hence the result follows from (1).

4.24. Theorem. (1) Let $0<a, b<c$ and $2 a b<c \leq a+b<c+1 / 2$. Then the function $f(r)=r^{\prime} \mathcal{K}(r)$ is strictly decreasing from $[0,1)$ onto $(0, B(a, b) / 2]$.

(2) Let $0<a, b<c<a+b$. Then the function $g(r)=r^{2(a+b-c)}(\mathcal{K}-\mathcal{E}) / r^{2}$ has positive Maclaurin coefficients and maps $(0,1)$ onto $(b B(a, b) /(2 c), B(c, a+b-c) / 2)$.

(3) Let $0<a, b<1, c=a+b$, and $a(2 b+1)<b+1<1 / a$. Then the function $h(r)=(\mathcal{K}-\mathcal{E}) / \log \left(1 / r^{\prime}\right)$ is decreasing from $(0,1)$ onto $(1, b B(a, b) / c)$. 
Proof. (1) Clearly $f(0)=B(a, b) / 2$ and by (3.9), $f(1)=0$. We have $f(r)=$ $g(r) / h(r)$, where

$$
g(r)=B(a, b) \sum_{0}^{\infty} \frac{(a, n)(b, n)}{(c, n) n !} r^{2 n}
$$

and

$$
h(r)=2 \sum_{0}^{\infty} d_{n} r^{2 n},
$$

with $d_{0}=1$ and $d_{n}=(1 \cdot 3 \cdot \ldots \cdot(2 n-1)) /(2 \cdot 4 \cdot \ldots \cdot(2 n))$ for $n=1,2,3, \ldots$. Hence, the coefficient ratio equals

$$
T_{n}=\frac{B(a, b)(a, n)(b, n) 2^{n}}{(c, n) \cdot 1 \cdot 3 \cdot \ldots \cdot(2 n-1)} .
$$

Then

$$
\frac{T_{n+1}}{T_{n}}=\frac{2(n+1)(n+b)}{(2 n+1)(n+c)}
$$

Now

$$
(2 n+1)(n+c)-2(n+a)(n+b)=n(2 c+1-2 a-2 b)+c-2 a b>0,
$$

so that $T_{n}$ is decreasing and hence by Theorem 4.3, $f$ is also decreasing.

(2) From (3.6), (3.9) and (4.16), we get

$$
f(r)=\frac{b B(a, b)}{2 c} r^{2(a+b-c)} F\left(a, b+1 ; c+1 ; r^{2}\right)=\frac{b B(a, b)}{2 c} F\left(c+1-a, c-b ; c+1 ; r^{2}\right) .
$$

Hence the assertion follows from (3.9).

(3) As in (2), from (3.6), (3.9) and (4.16), we get

$$
h(r)=\frac{b B(a, b)}{c} \frac{r^{2} F\left(a, b+1 ; c+1 ; r^{2}\right)}{2 \log \left(1 / r^{\prime}\right)} .
$$

Writing the Maclaurin series expansion of both the numerator and the denominator, the ratio of coefficients equals

$$
T_{n}=\frac{b B(a, b)}{c} \frac{(a, n)(b+1, n)(n+1)}{(c+1, n) n !}=\frac{B(a, b)(a, n)(b, n+1)(n+1)}{(c, n+1) n !} .
$$

Hence

$$
\frac{T_{n+1}}{T_{n}}=\frac{(a+n)(b+n+1)(n+2)}{(c+n+1)(n+1)^{2}} .
$$

Then,

$(c+n+1)(n+1)^{2}-(a+n)(b+n+1)(n+2)=n(1-a-a b)+(1+b-2 a b-a)>0$.

Hence, $T_{n}$ is decreasing, so that the result follows by (3.9) and Theorem 4.3,

4.25. Remark. Theorem 4.24(3) generalizes AQVV, Lemma 5.2 (12)]. The latter follows if we put $c=1$ in Theorem 4.24(3). 
4.26. Differential equations. The hypergeometric function $w=F(a, b ; c ; z)$ satisfies the differential equation [R, p. 54]

$$
z(1-z) w^{\prime \prime}+(c-(a+b+1) z) w^{\prime}-a b w=0 .
$$

Changing the variable $z$ to $z^{2}$, this reduces to

$$
z\left(1-z^{2}\right) w^{\prime \prime}+\left(2 c-1-(2 a+2 b+1) z^{2}\right) w^{\prime}-4 a b z w=0 .
$$

In particular, the generalized elliptic integrals, $w=\mathcal{K}_{a, c}(r)$ and $w=\mathcal{K}_{a, c}^{\prime}(r)$, satisfy, respectively, the differential equations

$$
r r^{\prime 2} w^{\prime \prime}+\left(2 c-1-(2 c+1) r^{2}\right) w^{\prime}-4 a(c-a) r w=0,
$$

and

$$
r r^{\prime 2} w^{\prime \prime}-\left(1-(2 c+1) r^{2}\right) w^{\prime}-4 a(c-a) r w=0 .
$$

In the special case $c=1$ the above two equations coincide (cf. AQVV, (4.3)], [L, 3.8.19, p. 75]). Next, the generalized elliptic integrals $w=\mathcal{E}_{a, c}(r)$ and $w=\mathcal{E}_{a, c}^{\prime}(r)$ satisfy, respectively, the differential equations

$$
r r^{\prime 2} w^{\prime \prime}-(2 c-1) r^{\prime 2} w^{\prime}+4(1-a)(c-a) r w=0,
$$

and

$$
r r^{\prime 2} w^{\prime \prime}-\left(1+(2 c-1) r^{2}\right) w^{\prime}+4(1-a)(c-a) r w=0 .
$$

In the special case $c=1$ the equations (4.29) and (4.30) are still different unlike in the case of (4.27) and (4.28) (cf. [L, 3.8.17, p. 74 and 3.8.23, p. 75]).

4.31. Correction. In AQVV, (4.3), p. 14] the first differential equation has a symmetry property, namely, it is satisfied both by $\mathcal{K}_{a}$ and $\mathcal{K}_{a}^{\prime}$. However, the second differential equation is satisfied only by $\mathcal{E}_{a}$, and not by $\mathcal{E}_{a}^{\prime}$. The differential equation satisfied by $w=\mathcal{E}_{a}^{\prime}$ is obtained by putting $c=1$ in (4.30). Thus it is

$$
r r^{\prime 2} w^{\prime \prime}-\left(1+r^{2}\right) w^{\prime}+4(1-a)^{2} r w=0 .
$$

We use the notation

$$
S_{w}=\left(\frac{w^{\prime \prime}}{w^{\prime}}\right)^{\prime}-\frac{1}{2}\left(\frac{w^{\prime \prime}}{w^{\prime}}\right)^{2}
$$

for the Schwarzian derivative.

4.32. Lemma. [R2, p. 9] Let $w_{1}, w_{2}$ be linearly independent solutions of the differential equation $w^{\prime \prime}+p(z) w^{\prime}+q(z) w=0$. Then $W=w_{1} / w_{2}$ satisfies the differential equation

$$
S_{W}(z)=2 q(z)-p^{\prime}(z)-p(z)^{2} / 2 .
$$


4.33. Theorem. Let $0<a, b<1$ and $2 c=a+b+1$. Then the modulus $\mu=\mu_{a, b, c}$ satisfies the differential equation

$$
S_{\mu}(r)=2 q(r)-p^{\prime}(r)-p(r)^{2} / 2,
$$

where

$$
p(r)=\frac{2 c-1-(4 c-1) r^{2}}{r r^{\prime 2}}, \quad q(r)=-\frac{4 a b}{r^{\prime 2}} .
$$

Proof. Follows immediately from Lemma 4.32 and (4.27).

4.34. Theorem. Let $0<a, b<1$ and $2 c=a+b$. Then the function $\nu=\mathcal{E}^{\prime} / \mathcal{E}$ satisfies the differential equation

$$
S_{\nu}(r)=2 q(r)-p^{\prime}(r)-p(r)^{2} / 2,
$$

where

$$
p(r)=\frac{2 c-1-(4 c-1) r^{2}}{r r^{\prime 2}}, \quad q(r)=-\frac{4(a-1) b}{r^{\prime 2}}
$$

Proof. Follows immediately from Lemma 4.32 and (4.29).

4.35. Lemma. If $0<a<\min \{c, 1\}$ and $c \leq a+(1 / 2)$, then the function $f(r)=$ $r \mathcal{K}_{a, c}(r) / \operatorname{arth} r$ is strictly decreasing from $(0,1)$ onto $(1, B / 2)$, where $B=B(a, c-a)$.

Proof. Let $f(r)=g(r) / h(r)$, where $g(r)=r \mathcal{K}_{a, c}(r)$ and $h(r)=\operatorname{arth} r$. Then $g(0)=h(0)=0$ and

$$
g^{\prime}(r) / h^{\prime}(r)=2(c-a) \mathcal{E}_{a, c}(r)+(1-2(c-a))\left(r^{\prime 2}\right) \mathcal{K}_{a, c}(r),
$$

which, by Lemma 4.21 (6), being a sum of two strictly decreasing functions, is also so. Hence, the monotonicity follows from L'Hôpital's Monotone Rule, Lemma 4.1. Finally, $f(0+)=B / 2$ by L'Hôpital's Rule and $f(1-)=g^{\prime}(1-) / h^{\prime}(1-)=1$, again by L'Hôpital's Rule.

Acknowledgments. The authors wish to thank the Finnish National Academy of Science and Letters, the Finnish Mathematical Society, and the Departments of Mathematics at the University of Helsinki and the University of Turku for generous support. The authors are indebted to Heikki Ruskeepää for providing expert help with the Mathematica ${ }^{\circledR}$ software. They are also grateful to D. Askey, B. C. Carlson, N. Stylianopoulos, and T. Sugawa for interesting discussions and correspondence. 


\section{References}

[AS] M. Abramowitz and I. A. Stegun, editors: Handbook of Mathematical Functions with Formulas, Graphs and Mathematical Tables, Dover, New York, 1965.

[Ah] L. V. Ahlfors: Complex Analysis, 2nd ed., McGraw-Hill, New York, 1966.

[AQVV] G. D. Anderson, S. -L. Qiu, M. K. Vamanamurthy, and M. Vuorinen: Generalized elliptic integrals and modular equations, Pacific J. Math. 192 (2000), 1-37.

[AVV] G. D. Anderson, M. K. Vamanamurthy, and M. Vuorinen: Conformal Invariants, Inequalities, and Quasiconformal Maps, J. Wiley, 1997.

[AAR] G. E. Andrews, R. Askey, And R. Roy: Special Functions, Cambridge Univ. Press, 1999.

[Ask] R. AskeY: Ramanujan and hypergeometric and basic hypergeometric series, Ramanujan Internat. Symposium on Analysis, December 26-28, 1987, ed. by N. K. Thakare, 1-83, Pune, India, Russian Math. Surveys 451 (1990), 37-86.

[Be] B. C. Berndt: Modular equations in Ramanujan's lost notebook, Number theory, 55-74, Trends Math., Birkhäuser, Basel, 2000.

[BBG] B. C. Berndt, S. Bhargava and F. G. Garvan: Ramanujan's theories of elliptic functions to alternative bases, Trans. Amer. Math. Soc., 347 (1995), 4163-4244.

[BCKZ] B. C. Berndt, H. H. Chan, S-Y. Kang, And L-C. Zhang: A certain quotient of eta-functions found in Ramanujan's lost notebook, Pacific J. Math. 202 (2002), no. 2, 267-304.

[BK] M. BIERNACKI AND J. KRZYŻ: On the monotonicity of certain functionals in the theory of analytic functions, Ann. Univ. M. Curie-Sklodowska, 2 (1955), 134-145.

[BB] J. M. Borwein And P. B. Borwein: Pi and the AGM, John Wiley \& Sons, New York, 1987.

[Bo] F. Bowman: Introduction to Elliptic Functions with applications, English Universities Press Ltd., London, 1953.

[BF] P. F. Byrd And M. D. Friedman: Handbook of Elliptic Integrals for Engineers and Scientists, 2nd ed., Grundlehren Math. Wiss. Vol. 67, Springer-Verlag, Berlin, 1971.

[CLT] H. H. Chan, W-C. Liaw AND V. TAN: Ramanujan's class invariant $\lambda_{n}$ and a new class of series for $1 / \pi$, J. London Math. Soc. (2) 64 (2001), no. 1, 93-106.

[DV] V. N. Dubinin And M. VuORInen: On conformal moduli of polygonal quadrilaterals, Preprint 417, August 2005, University of Helsinki, 12 pp.

[Hen] P. Henrici: Applied and Computational Complex Analysis, Vol. III, Wiley, New York, 1986.

[Her] J. HERSCH : On harmonic measures, conformal moduli and some elementary symmetry methods, J. Analyse Math. 42 (1982/83), 211-228.

[L] D. F. Lawden: Elliptic Functions and Applications, Applied Math. Sciences, Vol. 80, Springer-Verlag, New York, 1989. 
[LV] O. Lehto and K. I. Virtanen: Quasiconformal Mappings in the Plane, 2nd ed., Grundlehren Math. Wiss., Band 126, Springer-Verlag, New York, 1973.

[M] A. I. Markushevich: Theory of Functions of a Complex Variable, Vol. II, PrenticeHall, Englewood Cliffs, NJ, 1965.

[N] Z. Nehari: Conformal mapping, McGraw-Hill Book Co., Inc., New York, Toronto, London, 1952. viii +396 pp.

[PV] S. Ponnusamy and M. Vuorinen: Asymptotic expansions and inequalities for hypergeometric functions, Mathematika 44 (1997), 278-301.

[Q] S. L. QIU: Grötzsch ring and Ramanujan's modular equations, (Chinese) Acta Math. Sinica 43 (2000), no. 2, 283-290.

[QV1] S. L. QIU AND M. VuORINEn: Duplication inequalities for the ratios of hypergeometric functions, Forum Math. 12 (2000), no. 1, 109-133.

[QV2] S.-L. QIU AND M. VUORINEN: Landen inequalities for hypergeometric functions, Nagoya Math. J. 154 (1999), 31-56.

[R] E. D. Rainville: Special Functions, Macmillan, New York, 1960.

[R2] E. D. RAINVILLE: Intermediate differential equations, 2nd ed. Macmillan, New York, 1964.

[RV] A. Rasila And M. Vuorinen: Experiments with the moduli of quadrilaterals, Rev. Roumaine Math. Pures Appl. (to appear).

[S] L-C. Shen: On an identity of Ramanujan based on the hypergeometric series ${ }_{2} F_{1}(1 / 3,2 / 3 ; 1 / 2 ; x)$, J. Number Theory 69 (1998), no. 2, 125-134.

[WW] E. T. Whittaker and G. N. Watson: A Course of Modern Analysis, 4th ed., Cambridge Univ. Press, London, 1927.

\section{HEIKKALA}

SSH Communications Security Corp.

Valimotie 17

FIN-00380 Helsinki

Finland

email: ville.heikkala@ssh.com

fax: $+358-20-5007051$

\section{M.K. VAmanamurthy}

Department of Mathematics

The University of Auckland

P B 92019, Auckland

New Zealand

email: vamanamu@math.auckland.ac.nz

fax: +64-9-3737457 


\section{VUORINEN}

Department of Mathematics

FIN-20014 University of Turku

FINLAND

email: vuorinen@utu.fi

fax: +358-2-3336595 